\title{
MOCCA code for star cluster simulations - V. Initial globular cluster conditions influence on blue stragglers
}

\author{
Arkadiusz Hypki, ${ }^{1,2 \star}$ and Mirek Giersz ${ }^{2}$ \\ ${ }^{1}$ Leiden Observatory, Leiden University, PO Box 9513, NL-2300 RA Leiden, the Netherlands \\ ${ }^{2}$ Nicolaus Copernicus Astronomical Center, Bartycka 18, 00-716 Warsaw, Poland
}

Accepted XXX. Received YYY; in original form ZZZ

\begin{abstract}
The paper presents an analysis of properties of populations of blue stragglers (BSs) in evolving globular clusters, based on numerical simulations done with the Mocca code for various initial globular clusters conditions.

We find that various populations of BSs strongly depend on the initial semi-major axes distributions. With a significant number of compact binaries, the number of evolutionary BSs can be also significant. In turn, for semi-major axes distributions preferring binaries with wider orbits, dynamical BSs are the dominant ones. Their formation scenario is very distinct: for wide binaries the number of dynamical interactions is significantly larger. Most interactions are weak and increase only slightly the eccentricities. However, due to a large number of such interactions, the eccentricities of a number of binaries finally get so large that the stars collide.

We study how larger initial clusters' concentrations influence the BSs. Besides the expected increase of the number of dynamically created BSs (for denser GCs the probabilities of strong dynamical interactions and collisions are higher), we find that the number of the evolutionary BSs is not affected even by very high initial concentrations. This has a very important implication on observations - it supports the theory that the evolutionary BSs are the result of the unperturbed evolution of the primordial binaries.

In addition, the paper presents the evolution of the ratio between the number of BSs in binaries and as single stars $\left(R_{B / S}\right)$. For a vast diversity of models, the ratio $R_{B / S}$ approaches the value $\sim 0.4$. Additionally, we identified two subgroups which differ in the initial semi-major axes distributions. The first group starts with a high ratio $R_{B / S}$, it decreases with time and settles around 0.4 . The second group starts with lower values of the ratio $R_{B / S}$ and increases to about the same level 0.4. The first group is dominated by the evolutionary BSs originating from the semi-major axes distribution which create some number of compact binaries. In turn, the second group is dominated by the dynamical ones with the initial semi-major axes distribution preferring the wider binaries.

We find also that the initial eccentricity distributions seems to have a small or no influence on the population of BSs.
\end{abstract}

Key words: stellar dynamics - methods: numerical - globular clusters: evolution - stars: blue stragglers

\section{INTRODUCTION}

The subject of this paper concerns properties of blue straggler stars. They are particularly interesting today, because by studying these type of objects, one can get important constraints on the link between the stellar and dynamical evolution of star clusters. Star clusters are very efficient environments for creating such exotic objects.

^ E-mail: ahypki@strw.leidenuniv.nl
By studying them, one can reveal e.g. the dynamical history of a cluster and the role of dynamics on the stellar evolution. BSs properties can also provide some constraints for initial binary properties.

BSs are defined as stars that are brighter and bluer (hotter) than the main-sequence turn-off point (more than 2 mag above the turnoff point). These stars lie along an extension of the main-sequence (MS) in the Color Magnitude Diagram (CMD) and appear to be a rejuvenated stellar population. BSs are on the place in the CMD where they should already evolve away from the MS. Their mass is 


\section{Arkadiusz Hypki and Mirek Giersz}

larger that the turn-off mass and is of the order of $M=1.0-1.7 M_{\odot}$ (De Marco et al. 2005), which suggests some stellar merger or a mass transfer scenario for their creation. They were first discovered by Sandage (1953) in M3 and later observations showed that BSs are present essentially in all star clusters. (Piotto et al. 2004) observed 3000 BSs in 56 different size clusters. BSs were discovered also in open clusters, e.g. Mathieu \& Geller (2009) and dwarf galaxies e.g. Mateo et al. (1995), Mapelli et al. (2007) or Monelli et al. (2012)

\subsection{Channels of formation of blue stragglers}

Currently, there are two main scenarios considered as possible formation mechanisms for BSs. The first scenario is a mass transfer between binary companions which can possibly lead to the coalescence of the binary system (McCrea 1964; Zinn \& Searle 1976; Mateo et al. 1990; Pritchet \& Glaspey 1991; Knigge et al. 2009). The second leading scenario for creating BSs is a physical collision between stars (Hills \& Day 1976). Channels of formation combine together dynamical interactions between stars (collisions) and stellar evolution (mas transfer). However, the exact nature of channels of formation of these objects and their relative importance is still unclear. Moreover, there is still no observational mechanism able to distinguish BSs from both channels (first steps were however already made (Ferraro et al. 2006)).

According to Fusi Pecci et al. (1992), different environments could be responsible for different origins of BSs. In globular clusters which are not dense, BSs could form as evolutionary mergers of primordial binaries, while in high density GCs, BSs could form from dynamical interactions, particularly from interactions involving binaries. Recently more evidence appeared suggesting that these all scenarios are actually working simultaneously in the GCs (Ferraro et al. 1995, 1997, 2009).

The relative efficiency of these two main formation channels is still unknown. However, it is believed that they act with different efficiencies according to the cluster structural parameters (Fusi Pecci et al. 1992) and additionally they can work simultaneously in different radial parts of a star cluster (Ferraro et al. 1997; Mapelli et al. 2006). Particularly, the number of BSs formed in the cluster does not correlate with the predicted collision rate (Piotto et al. 2004; Leigh et al. 2007, 2008). This is one of the reasons why it is believed that mass transfer mechanisms are more important in the creation of BSs, instead of collisions between stars (Knigge et al. 2009). Unfortunately, there is still no simple observational distinction between BSs formation through mass transfer or collisions between stars. One of the first attempts to clarify this issue is the approach of Ferraro \& Lanzoni (2009), who observed a significant depletion of $\mathrm{C}$ and $\mathrm{O}$ suggesting mass transfer mechanisms for creating some BSs subpopulations in 47 Tuc. According to Davies et al. (2004) primordial binaries with BSs are vulnerable to exchange encounters in the crowded environments of star clusters. Low-mass components are replaced by more massive single stars. The authors claim that these encounters tend to reduce the number of binaries containing primaries with masses close to the present turn-off mass. Thus, the population of primordial BSs is reduced in more massive star clusters.

\subsection{Masses of blue stragglers}

First estimates of masses of BSs were performed by Shara et al. (1997). They performed direct measurements of BSs in 47 Tuc GC where they used spectroscopic analysis of HST data. They derived the mass of $M=1.7 M_{\odot}$, which is twice as large as the turn-off mass for 47 Tuc. Later, De Marco et al. (2005) calculated masses for 4 BSs $\left(1.27,1.0 .5,0.99\right.$ and $0.99 M_{\odot}$ ) but with slightly larger errors and thus evolutionary tracks did not have a very good agreement with BSs masses. In turn, very precise masses determinations were performed by using spectroscopic and photometric analysis of eclipsing binaries, Thompson et al. (2010) for 47 Tuc, Kaluzny et al. (2007, a), Kaluzny et al. (2007, b), Kaluzny et al. (2009) for 47 Tuc, $\omega$ Cen and NGC 6752. The last papers showed a very good agreement between estimated BSs masses and predicted masses from single-star evolutionary tracks. However, there are examples of works which studied BSs in binaries and found that single-star evolutionary models overestimate the dynamical masses. For example Geller \& Mathieu (2012) gives values of overestimation of $15-30 \%$ for BSs in NGC 188 ( 7 Gyr old).

Lanzoni et al. (2007) determined masses for 34 BSs for NGC 1904 using theoretical isochrones and trying to fit them to the photometric data in $\mathrm{V}$ and $\mathrm{B}-\mathrm{V}$ colors. The metallicity was chosen to be $Z=6 \times 10^{-4}$ (33 times smaller than solar metallicity) and the reddening $E(B-V)=0.01$ (Ferraro et al. 1999). The isochrone for the age of $12 \mathrm{Gyr}$ reproduced the main sequence nicely, while isochrones for BSs were calculated for ages of 16 Gyr, with 0.5 Gyr step, which covered the whole BSs population on CMD. The computed isochrones created a mesh of possible evolutionary tracks. For all BSs colors and magnitudes of the closest evolutionary track were chosen, and after a simple projection the masses for BSs were derived. They are in the range range from $\sim 0.95$ to $\sim 1.6 M_{\odot}$, the mean and median BS mass is $1.2 M_{\odot}$ and the turn-off mass is estimated to be $M_{\text {turn-off }}=0.8 M_{\odot}$.

Blue stragglers masses can be also calculated based on the pulsation properties (Fiorentino et al. 2014). They used HST images to study the population of variable BSs in the central region of NGC 6541. NGC 6541 is an old GC 13.25 \pm 1 Gyr (Dotter et al. 2010), metal poor $[\mathrm{Fe} / \mathrm{H}]=-1.76 \pm-0.02$ (Lee \& Carney 2002), $3 \mathrm{kpc}$ from the center of the Milky Way, and it is a dynamically old, classified as post core-collapse cluster (Harris 1996). Among all BSs Fiorentino et al. (2014) discovered three W UMa and nine SX Phoenicis stars (SXP). SXP stars cross the faint extension of the classical instability strip (IS, see e.g. Pych et al. (2001)). IS is a place on the Hertzsprung-Russell diagram (HR) where pulsating, constantly expanding and contracting, stars are located due to imbalance of their thermal pressure and the gravitational force. SXP show a photometric variability on very short scales with periods $P \lesssim 0.1$ [d] and can be unstable for radial and nonradial pulsations. However, these variables follow the classical pulsation equation, relating the observed period to the intrinsic stellar parameters such as mass, luminosity, and effective temperature $P\left(M, L, T_{e f f}\right)$, for any given pulsation mode and chemical composition. Thus, this equation can be used to estimate SXP masses. For SXP stars periods, mean magnitudes and pulsation modes were obtained and masses were calculated using pulsation equations obtained from linear nonadiabatic models (Santolamazza et al. 2001). The masses are in the range $1.0-1.1 M_{\odot}$, which is significantly larger than the main sequence turn-off mass $\left(\sim 0.75 M_{\odot}\right)$. The computed masses turned out to be in agreement with evolutionary tracks for single stars (Fiorentino et al. 2014). The SXP stars are of the great importance because if they e.g. pulsate in the fundamental modes they can be used as "standard candles" for precise distance calculations (e.g. Otulakowska et al. (2011) for NGC 2155). 


\subsection{Other blue straggler properties}

Sollima et al. (2008) tested 13 low-density GCs for correlations between the specific frequency of BSs and cluster parameters like binary fraction, total magnitude, age, central velocity dispersion, metallicity, cluster central density, half-mass relaxation time, halfmass radius, stellar collision rate, concentration, and cluster evaporation rate. The BSs specific frequency was defined as the ratio between the estimated BSs number and MS number. MSs were chosen, instead of horizontal branch (HB) or red giant branch (RGB) stars, because of their abundance in all clusters and their completeness. They found the strongest correlation between the number of BSs and the binary fraction. It suggests that the primordial binary fraction is one of the most important factor for producing BSs. Additionally, a noticeable correlation exists with the absolute magnitude and an anticorrelation with the cluster age and central velocity dispersion. The age estimates are uncertain and span a narrow range, so one has to be careful while making any generalizations. However, if such anticorrelation with the cluster ages is confirmed in the future, it could suggest that binary disruptions in cores of GCs become more efficient with time, which would in consequence reduce the fraction of binaries and also BSs in the core. Sollima et al. (2008) suggest that the strong correlation between the number of BSs and the binary fraction is a result of the formation channel of BSs in the form of unperturbed evolution of primordial binary systems. They found no correlations for central density, concentration, stellar collision rate and half-mass relaxation time. This indicates that the collisional channel of BSs formation has a very small efficiency in low-density GCs.

Finding an observational mechanism to determine the channel of formation of a BS is very important. It could provide valuable boundaries for the processes which lead to their creation. It would also help to investigate how different channels of formation of BSs depend on the properties of GCs. Ferraro et al. (2006) gave the first results of the chemical composition of BSs for some selected GCs. They examined 43 BSs in 47 Tuc and found the first evidence that some subpopulations of these BSs have significant depletion of $\mathrm{C}$ and $\mathrm{O}$ with respect to the normal cluster stars. They argue that this is caused by CNO burning products on the BS surface, coming from the core of a deeply peeled primary star. This scenario is expected for the case of the mass transfer formation mechanism and could be the first direct proof of this formation process. Later, Fossati et al. (2010) attempted to develop a formation scenario for HD 73666, a known BS from the Praesepe cluster, and showed that the abundance of $\mathrm{CNO}$ is consistent with a collisional formation. However, they were unable to determine whether HD 73666 is a product of a collision between two stars, components of a binary, or between binary systems. Further studies of these phenomena could create some statistics on how efficient this mechanism could be in producing BSs.

Ferraro et al. (2009) reported two distinct sequences of BSs in GC M30. These two groups are clearly separated in the CMD and nearly parallel to each other (Ferraro et al. 2009, Fig. 1). The first BSs sequence was accurately reproduced by the collisional isochrones (Ferraro et al. 2009, Fig. 4, blue points). The second BSs sequence corresponds well to the zero-age main sequence shifted by $0.75 \mathrm{mag}$, marking the position of the low-luminosity boundary predicted for a population of mass-transfer binary systems (Ferraro et al. 2009, Fig. 4, red points).

Knigge et al. (2009) focused on BSs in cores of star clusters, because in these regions collisions between stars should be frequent. They used existing data from a large set of HST-based CMDs and confirmed that there is no global correlation between the observed core BSs number and the collision rate (different core densities have different predicted collision rates and it does not correlate with the number of BSs). However, there is a significant correlation if one would restrict this relation to the clusters with dense cores (see Knigge et al. (2009) black points in Fig. 1). The second relation which was tested by this group concerns the binary fraction in the core. If most of BSs were formed in binaries, the number of BSs should scale with the binary fraction simply as $N_{B S S} \propto f_{\text {bin }} M_{\text {core }}$, where $f_{\text {bin }}$ is the binary fraction in the core, and $M_{\text {core }}$ is the total stellar mass contained in the core. Indeed, they found a clear correlation between the number of BSs and core masses of the clusters, as it is expected for the scenario where most BSs originate from binaries (see Knigge et al. (2009) Fig. 2). They interpret this result as a strong evidence that more BSs originates from binaries instead of collisions between stars. They found that the dependence $N_{B S S} \propto M_{\text {core }}^{\delta}$ can be estimated with $\delta \simeq 0.4-0.5$. Furthermore, they estimated the power-law correlation $f_{\text {bin }} \propto M_{\text {core }}^{-0.35}$ based on the data from Milone et al. (2008) who described global parameters for 35 clusters spanning a wide range of density and other dynamical star cluster parameters. Those two estimates combined together shows that the number of BSs found in the cores of GCs scales roughly as $N_{B S S} \propto f_{\text {bin }} M_{\text {core }}$, just as expected if most core BSs are formed in binary systems (Knigge et al. 2009).

BSs are being found in the halo and in the bulge of the Galaxy (Bragaglia et al. 2005; Fuhrmann et al. 2011; Clarkson et al. 2011). Tillich et al. (2010) found a BS from the halo which has a radial velocity of about $504.6 \pm 5 \mathrm{~km} / \mathrm{s}$. With a Galactic rest-frame velocity of about $467 \mathrm{~km} / \mathrm{s}$, it makes this BS one of the fastest moving BSs (but it is still bound to the Galaxy).

Recently, Geller \& Mathieu (2011) reported that BSs in longperiod binaries in an old (7 Gyr) open cluster, NGC 188, have companions with masses of about half of the solar mass, which is a surprisingly narrow mass distribution. This rules out the collisional origin for these long-period BSs, because otherwise, for the collision hypothesis, there would be significantly more companions with higher masses. The data is consistent with a mass transfer origin for the long-period blue straggler binaries in NGC 188, in which the companions would be white dwarfs of about half of the solar mass.

This paper is organized as follows. In the Sect. 2 there is shortly described the MOCCA code, summary of performed numerical simulations and description of the data analysis methods. Sect. 3 contains the detailed analysis how various initial conditions influence on the population of BSs. Additionally, we discuss how the ratio of BSs in binaries and as single stars changes for various models. Finally, Sect. 4 summarizes our findings and presents discussion about channels of formation of BSS.

\section{NUMERICAL SIMULATIONS}

Numerical simulations were performed with the $\operatorname{moccs}^{1}$ code (Hypki \& Giersz 2013).

The mocca code is currently one of the most advanced codes which is able to simulate real size GCs and at the same time, it allows to have a full dynamical history of the evolution of all stars in the system. MоссA is an improved version of the Monte Carlo code, originally developed by Hénon (1971), improved by Stodolkiewicz

\footnotetext{
${ }^{1} \mathrm{http}: / /$ moccacode.net
} 
(1986), and finally heavily developed by Giersz and his collaborators (Giersz 1998, 2001, 2006; Giersz et al. 2011, 2013). The мосca code combines together the old version of the code (Monte Carlo method) with strong dynamical interactions performed with the Fewbody code (Fregeau \& Rasio 2007). The stellar evolution is done for both single and binary stars using SSE and BSE codes (Hurley et al. 2000, 2002). All these codes together create one package, called the Mocca code, which stands for MOnte Carlo Cluster simulAtor (for detail description of the mocca code see Hypki \& Giersz (2013)).

The speed of the mocca code is its greatest advantage in comparison to N-body codes. During the same amount of time one can run multiple simulations with the Mocca code to cover a very wide range of initial cluster parameters. Instead of having one simulation from N-body code, one can have hundreds of simulations from the MOcCA code and one can perform detailed statistical analysis of the results. Additionally, mocca simulations give practically the same amount of information about the evolution of star clusters as $\mathrm{N}$ body codes, which makes it even more attractive. There is already number of papers, which shows this agreement across all previous version of the Mocca code (Giersz \& Heggie 1994a,b, 1996, 1997; Giersz \& Spurzem 1994), and especially with the current version Giersz et al. (2013); Wang et al. (2016).

\subsection{Initial parameters for the мосса code simulations}

For the purpose of this paper a large number of simulations was computed. These simulations vary in many aspects. They have different initial mass functions, binary properties, different sizes, concentrations (thus different scales of the dynamical evolution), and more. The purpose of computing many simulations was to check how different properties of blue stragglers depend on the initial conditions of GCs and distributions of initial binary properties.

The mocca code allows to define many different initial conditions. However, only a subset of them was used. The chosen parameters are believed to be the ones which could have the biggest influence on the population of the BSs. The simulations, together with their initial conditions, are summarized in the Tab. 1 and Tab. 2.

A majority of the models have $20 \%$ of the primordial binaries. Typically, GCs contain at a present time $\sim 5-10 \%$ of binaries. We decided to start the models with a higher value to simply have a larger number of BSs, in order to make some features easier to notice. All models use only the Plummer model as a density distribution. We decided to use it, instead of the King models, because it is simple and accurate enough for the initial mass distribution.

Tables Tab. 1 and Tab. 2 contain over 60 models. The models from Tab. 1 differ mainly in the values of initial number of stars, tidal radii $\left(r_{t i d}\right)$, and concentrations $\left(c=r_{\text {tid }} / r_{h}\right)$. These are the parameters which define GCs with different dynamical scales, from slowly evolving models, up to models with fast dynamical evolution. Various dynamical scales of these models should have a different influence on the spatial distribution of BSs in GCs, which is essential for the studies of the formation of the bimodal spatial distribution observed in many real GCs. Thus, the models from Tab. 1 (from моссA-1 up to MoсcA-43) were mainly used to study the spatial distributions of BSs in evolving GCs (see Hypki (2016), MOCCA code for star cluster simulations - VI. Bimodal spatial distribution of blue stragglers, submitted). However, these models were also used to study the relation between the number of BSs in binaries and as single stars (see Sect. 3.3).

The models with identifiers larger than 43 (Tab. 2) were mainly used to study how different initial binary conditions influ- ence the population of BSs of different types. They have different mass ratios for components in binaries, different distributions of semi-major axes and eccentricities but the same initial number of stars and concentrations (except Mocca-63). The diversity of initial properties of binaries allows to study how the number of BSs from different channels depends on the initial conditions.

All models from Tab. 1 and Tab. 2 were used to study the ratio between BSs in binaries and as single stars (see Sect. 3.3). Different sizes and concentrations, as well as different initial properties of binaries, are expected to have an influence on this ratio.

The models from Tab. 1 and Tab. 2 are only a small subset of the models actually computed. The total number of models was much higher and concerned even broader range of initial conditions. However, the models from these two tables compose a complete subset of models which is sufficient enough to support conclusions stated in this paper.

From this point any core radius $\left(r_{c}\right)$ refers to this calculated according to Casertano \& Hut (1985), and relaxation time $\left(t_{r h}\right)$ refers to the half-mass relaxation time unless it is noted otherwise. BS is detected in the Mocca code if it exceeds the turn-off mass by at least $2 \%$ (to be consistent with the first results on BSs presented by Hypki \& Giersz (2013)).

\subsection{Data analysis}

Data analysis of the results of the Mocca code is very challenging. The output files are large. One simulation with 600k initial stars can easily exceed a few GBs. When there are several dozens of simulations, the analysis of such large data sets is not trivial.

Each MосcA simulation contains almost 20 different files. Each file stores different kind of data. For many cases querying the data is simple - it is just the extraction and visualization of a few columns. However, real life queries are much more complicated. In the analysis there is often a need to read data from many files simultaneously in order to prepare meaningful results. If the same procedure has to be applied for many simulations, the overall complexity of data analysis increases significantly. Thus, for the data analysis of the results of the MоссA code there were created many scripts which simplify this process.

All scripts for data analysis are written in Java. They share the same core library, which means that the process of building next scripts is significantly simplified. The scripts are built with Object Oriented Programming (OOP) paradigm in mind. It means that it consists of small Java classes responsible for small tasks. By combining them into larger Java classes, one can create a modular code able to solve complex tasks while still being easy to understand and change. OOP programming is especially useful for the data analysis of the mосса simulations because each entity from the мосса code, like star or binary, can be expressed as one Java class. Each Java class can have an arbitrary number of properties. In the case of мосса, they are for instance: mass, radius, luminosity of stars, and semi-major axis and eccentricity of binaries. In this way one can create very clean and fast scripts to analyze many моссA simulations.

The output from the mocca code was split into a number of files. Each file contains only one type of information. Some of them store information on global parameters of GC, positions and velocities of stars (data on dynamics), interactions between two binaries or binaries with single stars, stellar evolution etc. What is more, such output divided into separate files is much easier to maintain and to understand by new users of the Mocca code.

The scripts allowed to optimize the disk usage of the MосcA 


\begin{tabular}{|c|c|c|c|c|c|c|c|c|c|c|c|}
\hline \multicolumn{12}{|c|}{ INITIAL MASS FUNCTION OF THE MOCCA SIMULATIONS (PART I) } \\
\hline Name & $N$ & $f_{b}$ & IM & $I M F_{s}$ & $I M F_{b}$ & $\mathrm{q}$ & a & $\mathrm{e}$ & $\mathrm{z}$ & $r_{t i d}$ & $r_{h}$ \\
\hline MOCCA-1 & $300 \mathrm{k}$ & 0.1 & $\mathrm{P}$ & K93 & K91 & $\mathrm{U}$ & $\mathrm{UL}$ & $\mathrm{T}$ & 0.001 & 69 & 6.9 \\
\hline MOCCA-2 & $300 \mathrm{k}$ & 0.2 & $\mathrm{P}$ & K93 & K91 & $\mathrm{U}$ & UL & $\mathrm{T}$ & 0.001 & 15 & 1.5 \\
\hline MOCCA-3 & $300 \mathrm{k}$ & 0.2 & $\mathrm{P}$ & K93 & K91 & $\mathrm{U}$ & UL & $\mathrm{T}$ & 0.001 & 25 & 2.5 \\
\hline MOCCA-4 & $300 \mathrm{k}$ & 0.2 & $\mathrm{P}$ & K93 & K91 & $\mathrm{U}$ & UL & $\mathrm{T}$ & 0.001 & 35 & 3.5 \\
\hline MOCCA-5 & $300 \mathrm{k}$ & 0.2 & $\mathrm{P}$ & K93 & K91 & $\mathrm{U}$ & $\mathrm{UL}$ & $\mathrm{T}$ & 0.001 & 45 & 4.5 \\
\hline MOCCA-6 & $300 \mathrm{k}$ & 0.2 & $\mathrm{P}$ & K93 & K91 & $\mathrm{U}$ & UL & $\mathrm{T}$ & 0.001 & 69 & 1.2 \\
\hline MOCCA-7 & $300 \mathrm{k}$ & 0.2 & $\mathrm{P}$ & K93 & K91 & $\mathrm{U}$ & $\mathrm{UL}$ & $\mathrm{T}$ & 0.001 & 69 & 1.7 \\
\hline MOCCA-8 & $300 \mathrm{k}$ & 0.2 & $\mathrm{P}$ & K93 & K91 & $\mathrm{U}$ & UL & $\mathrm{T}$ & 0.001 & 69 & 2.3 \\
\hline MOCCA-9 & $300 \mathrm{k}$ & 0.2 & $\mathrm{P}$ & K93 & K91 & $\mathrm{U}$ & UL & $\mathrm{T}$ & 0.001 & 69 & 2.8 \\
\hline MOCCA-10 & $300 \mathrm{k}$ & 0.2 & $\mathrm{P}$ & K93 & K91 & $\mathrm{U}$ & $\mathrm{UL}$ & $\mathrm{T}$ & 0.001 & 69 & 3.5 \\
\hline MOCCA-11 & $300 \mathrm{k}$ & 0.2 & $\mathrm{P}$ & K93 & K91 & $\mathrm{U}$ & UL & $\mathrm{T}$ & 0.001 & 69 & 4.6 \\
\hline MOCCA-12 & $300 \mathrm{k}$ & 0.2 & $\mathrm{P}$ & K93 & K91 & $\mathrm{U}$ & UL & $\mathrm{T}$ & 0.001 & 69 & 6.9 \\
\hline MOCCA-13 & $300 \mathrm{k}$ & 0.2 & $\mathrm{P}$ & K93 & K91 & $\mathrm{U}$ & UL & $\mathrm{T}$ & 0.001 & 69 & 9.9 \\
\hline MOCCA-14 & $300 \mathrm{k}$ & 0.2 & $\mathrm{P}$ & K93 & K91 & $\mathrm{U}$ & UL & $\mathrm{T}$ & 0.001 & 69 & 17.3 \\
\hline MOCCA-15 & $300 \mathrm{k}$ & 0.2 & $\mathrm{P}$ & K93 & K91 & $\mathrm{U}$ & UL & $\mathrm{T}$ & 0.001 & 85 & 8.5 \\
\hline MOCCA-16 & $300 \mathrm{k}$ & 0.2 & $\mathrm{P}$ & K93 & K91 & $\mathrm{U}$ & $\mathrm{UL}$ & $\mathrm{T}$ & 0.001 & 135 & 13.5 \\
\hline MOCCA-17 & $300 \mathrm{k}$ & 0.2 & $\mathrm{P}$ & K93 & K91 & $\mathrm{U}$ & UL & $\mathrm{T}$ & 0.001 & 235 & 23.5 \\
\hline MOCCA-18 & $300 \mathrm{k}$ & 0.2 & $\mathrm{P}$ & K93 & K91 & $\mathrm{U}$ & UL & $\mathrm{T}$ & 0.001 & 335 & 33.5 \\
\hline MOCCA-19 & $300 \mathrm{k}$ & 0.3 & $\mathrm{P}$ & K93 & K91 & $\mathrm{U}$ & UL & $\mathrm{T}$ & 0.001 & 69 & 9.6 \\
\hline MOCCA-20 & $300 \mathrm{k}$ & 0.5 & $\mathrm{P}$ & K93 & K91 & $\mathrm{U}$ & UL & $\mathrm{T}$ & 0.001 & 69 & 9.6 \\
\hline MOCCA-21 & $600 \mathrm{k}$ & 0.05 & $\mathrm{P}$ & K93 & K91 & $\mathrm{U}$ & UL & $\mathrm{T}$ & 0.001 & 100 & 10.0 \\
\hline MOCCA-22 & $600 \mathrm{k}$ & 0.1 & $\mathrm{P}$ & K93 & K91 & $\mathrm{U}$ & $\mathrm{UL}$ & $\mathrm{T}$ & 0.001 & 100 & 10.0 \\
\hline MOCCA-23 & $600 \mathrm{k}$ & 0.2 & $\mathrm{P}$ & K93 & K91 & $\mathrm{U}$ & UL & $\mathrm{T}$ & 0.001 & 25 & 2.5 \\
\hline MOCCA-24 & $600 \mathrm{k}$ & 0.2 & $\mathrm{P}$ & K93 & K91 & $\mathrm{U}$ & UL & $\mathrm{T}$ & 0.001 & 35 & 0.9 \\
\hline MOCCA- 25 & $600 \mathrm{k}$ & 0.2 & $\mathrm{P}$ & K93 & K91 & $\mathrm{U}$ & UL & $\mathrm{T}$ & 0.001 & 35 & 1.2 \\
\hline MOCCA-26 & $600 \mathrm{k}$ & 0.2 & $\mathrm{P}$ & K93 & K91 & $\mathrm{U}$ & UL & $\mathrm{T}$ & 0.001 & 35 & 1.8 \\
\hline MOCCA- 27 & $600 \mathrm{k}$ & 0.2 & $\mathrm{P}$ & K93 & K91 & $\mathrm{U}$ & UL & $\mathrm{T}$ & 0.001 & 35 & 3.5 \\
\hline MOCCA- 28 & $600 \mathrm{k}$ & 0.2 & $\mathrm{P}$ & K93 & K91 & $\mathrm{U}$ & UL & $\mathrm{T}$ & 0.001 & 55 & 1.4 \\
\hline MOCCA-29 & $600 \mathrm{k}$ & 0.2 & $\mathrm{P}$ & K93 & K91 & $\mathrm{U}$ & $\mathrm{UL}$ & $\mathrm{T}$ & 0.001 & 55 & 1.8 \\
\hline MOCCA-30 & $600 \mathrm{k}$ & 0.2 & $\mathrm{P}$ & K93 & K91 & $\mathrm{U}$ & UL & $\mathrm{T}$ & 0.001 & 55 & 2.8 \\
\hline MOCCA-31 & $600 \mathrm{k}$ & 0.2 & $\mathrm{P}$ & K93 & K91 & $\mathrm{U}$ & UL & $\mathrm{T}$ & 0.001 & 55 & 5.5 \\
\hline MOCCA-32 & $600 \mathrm{k}$ & 0.2 & $\mathrm{P}$ & K93 & K91 & $\mathrm{U}$ & UL & $\mathrm{T}$ & 0.001 & 100 & 1.7 \\
\hline MOCCA-33 & $600 \mathrm{k}$ & 0.2 & $\mathrm{P}$ & K93 & K91 & $\mathrm{U}$ & UL & $\mathrm{T}$ & 0.001 & 100 & 2.5 \\
\hline MOCCA-34 & $600 \mathrm{k}$ & 0.2 & $\mathrm{P}$ & K93 & K91 & $\mathrm{U}$ & UL & $\mathrm{T}$ & 0.001 & 100 & 5.0 \\
\hline MOCCA-35 & $600 \mathrm{k}$ & 0.2 & $\mathrm{P}$ & K93 & K91 & $\mathrm{U}$ & $\mathrm{UL}$ & $\mathrm{T}$ & 0.001 & 100 & 10.0 \\
\hline MOCCA-36 & $600 \mathrm{k}$ & 0.2 & $\mathrm{P}$ & K93 & K91 & $\mathrm{U}$ & UL & $\mathrm{T}$ & 0.001 & 100 & 20.0 \\
\hline MOCCA-37 & $600 \mathrm{k}$ & 0.2 & $\mathrm{P}$ & K93 & K91 & $\mathrm{U}$ & $\mathrm{UL}$ & $\mathrm{T}$ & 0.001 & 180 & 18.0 \\
\hline MOCCA-38 & $600 \mathrm{k}$ & 0.2 & $\mathrm{P}$ & K93 & K91 & $\mathrm{U}$ & $\mathrm{UL}$ & $\mathrm{T}$ & 0.001 & 130 & 13.0 \\
\hline MOCCA-39 & $600 \mathrm{k}$ & 0.2 & $\mathrm{P}$ & K93 & K91 & $\mathrm{U}$ & UL & $\mathrm{T}$ & 0.001 & 230 & 23.0 \\
\hline MOCCA -40 & $600 \mathrm{k}$ & 0.2 & $\mathrm{P}$ & K93 & K91 & $\mathrm{U}$ & $\mathrm{UL}$ & $\mathrm{T}$ & 0.001 & 300 & 30.0 \\
\hline MOCCA-41 & $600 \mathrm{k}$ & 0.2 & $\mathrm{P}$ & K93 & K91 & $\mathrm{U}$ & UL & $\mathrm{T}$ & 0.001 & 400 & 40.0 \\
\hline MOCCA-42 & $600 \mathrm{k}$ & 0.4 & $\mathrm{P}$ & K93 & K91 & $\mathrm{U}$ & $\mathrm{UL}$ & $\mathrm{T}$ & 0.001 & 100 & 10.0 \\
\hline MOCCA-43 & $600 \mathrm{k}$ & 0.5 & $\mathrm{P}$ & K93 & K91 & $\mathrm{U}$ & UL & $\mathrm{T}$ & 0.001 & 100 & 10.0 \\
\hline
\end{tabular}

Table 1. Initial conditions of Mocca simulations done for the purpose of this paper. Symbols have the following meaning: $\mathrm{N}-$ initial number of objects (single + binary stars), $f_{b}$ - initial binary fraction, $f_{b}=N_{b} / N$ ( $N_{b}$ - number of binaries), IM - initial model, $\mathrm{P}-\mathrm{Plummer}$ model, $I M F_{s}-$ Initial Mass Function for single stars, K93 - Kroupa et al. (1993) in the range [0.1; 100]M $\odot, I M F_{b}$ - Initial Mass Function for binary stars, K91 - Kroupa et al. (1991, eq. 1), binary masses from 0.2 to $100 \mathrm{M}_{\odot}, \mathrm{q}$ - distribution of mass ratios between stars in binaries, $\mathrm{U}$ - uniform distribution of mass ratios, $\mathrm{R}$ - random pairing of masses for binary components, a - semi-major axes distribution, $\mathrm{UL}-$ uniform distribution of semi-major axes in the logarithmic scale from $2\left(R_{1}+R_{2}\right)$ to $100 \mathrm{AU}, \mathrm{L}$ - lognormal distribution of semi-major axes from $2\left(R_{1}+R_{2}\right)$ to $100 \mathrm{AU}, \mathrm{K} 95$ - binary period distribution from Kroupa (1995a), K95E - distribution of semi-major axes with eigenevolution and feeding algorithm (Kroupa 1995a), K13 - new eigenevolution and feeding algorithm (Kroupa et al. 2013), e eccentricity distribution, $\mathrm{T}-$ thermal eccentricity distribution, $\mathrm{TE}-$ thermal eccentricity distribution with eigenevolution, $\mathrm{z}-$ mettalicity $(\mathrm{e} . \mathrm{g}$. $0.001=1 / 20$ of the solar metallicity 0.02$), r_{\text {tid }}$ - tidal radius in $\mathrm{pc}, r_{h}$ - half-mass radius in $\mathrm{pc}$

simulations. The largest file which is produced is a snapshot file, which contains the full image of a GC with a number of parameters for each star (in total 30 parameters per object). The snapshots are produced usually every 50 or $200 \mathrm{Myr}$. Thus, the output file becomes very large (even $>20 \mathrm{GBs}$ for one simulation). In order to save disk space, a more advanced solution was implemented. The snapshot can be saved in a compact form with only 4 values: ID, position, radial and tangential velocities - the only values which are not stored in other output files. All the other properties, like masses, radii, semi-major axes, one can recreate from other output files (e.g. from files storing data of stellar evolution or dynamical interactions). The script automatically detects whether a snapshot is in the compact form or in a default mode (with all columns). If the snapshot is compact, then the scripts can automatically rebuild full snapshot. Additionally, all output files can be compressed using gzip algorithm and thus saving even more disk space. The scripts handle compressed data on-the-fly as well. All these efforts made the need for disk space decrease a lot. It is especially useful for Big Survey project, which goal is to produce and maintain thousands of MoccA simulations of real size GCs for the vast mesh of initial 


\begin{tabular}{|c|c|c|c|c|c|c|c|c|c|c|c|}
\hline & & & & & & & ATI & & & & \\
\hline Name & $N$ & $f_{b}$ & IM & $I M F_{s}$ & $I M F_{b}$ & $\mathrm{q}$ & a & $\mathrm{e}$ & $\mathrm{z}$ & $r_{\text {tid }}$ & $r_{h}$ \\
\hline мосСА- 44 & $300 \mathrm{k}$ & 0.2 & $\mathrm{P}$ & K93 & K91 & $\mathrm{R}$ & UL & $\mathrm{T}$ & 0.001 & 69 & 6.9 \\
\hline MOCCA-45 & $300 \mathrm{k}$ & 0.2 & $\mathrm{P}$ & K9? & K91 & $\mathrm{R}$ & $\mathrm{L}$ & $\mathrm{T}$ & 0.001 & 69 & 6.9 \\
\hline моссA- 46 & $300 \mathrm{k}$ & 0.2 & $\mathrm{P}$ & K93 & K91 & $\mathrm{R}$ & K95 & $\mathrm{T}$ & 0.001 & 69 & 6.9 \\
\hline MOCCA-47 & $300 \mathrm{k}$ & 0.2 & $\mathrm{P}$ & K93 & K91 & $\mathrm{R}$ & K95E & $\mathrm{T}$ & 0.001 & 69 & 6.9 \\
\hline мосса -48 & $300 \mathrm{k}$ & 0.2 & $\mathrm{P}$ & K93 & K91 & $\mathrm{R}$ & K13 & $\mathrm{T}$ & 0.001 & 69 & 6.9 \\
\hline MOССA- 49 & $300 \mathrm{k}$ & 0.2 & $\mathrm{P}$ & $\mathrm{KO}$ & K9 & $\mathrm{R}$ & UL & $\mathrm{TE}$ & 0.001 & 69 & 6.9 \\
\hline MOCCA -50 & $300 \mathrm{k}$ & 0.2 & $\mathrm{P}$ & K93 & $\mathrm{Kg}$ & $\mathrm{R}$ & $\mathrm{L}$ & $\mathrm{TE}$ & 0.001 & 69 & 6.9 \\
\hline MOCCA -51 & $300 \mathrm{k}$ & 0.2 & $\mathrm{P}$ & & & $\mathrm{R}$ & K95 & $\mathrm{TE}$ & 0.001 & 69 & 6.9 \\
\hline MOССA-52 & $300 \mathrm{k}$ & 0.2 & $\mathrm{P}$ & K & K & $\mathrm{R}$ & K95E & $\mathrm{TE}$ & 0.00 & 69 & 6.9 \\
\hline MOCCA -53 & $300 \mathrm{k}$ & 0.2 & $\mathrm{P}$ & K & K & $\mathrm{R}$ & K13 & $\mathrm{TE}$ & 0.0 & 69 & 6.9 \\
\hline MOCCA -54 & $300 \mathrm{k}$ & 0.2 & $\mathrm{P}$ & K & K91 & $\mathrm{U}$ & $\mathrm{L}$ & $\mathrm{T}$ & 0.0 & 69 & 6.9 \\
\hline MOССA -55 & $300 \mathrm{k}$ & 0.2 & $\mathrm{P}$ & K9 & K9 & $\mathrm{U}$ & K95 & 1 & 0.001 & 69 & 6.9 \\
\hline MOCCA-56 & $300 \mathrm{k}$ & 0.2 & $\mathrm{P}$ & K9 & K9 & $\mathrm{U}$ & K95E & $\mathrm{T}$ & 0.001 & 69 & 6.9 \\
\hline MOССA-57 & $300 \mathrm{k}$ & 0.2 & $\mathrm{P}$ & K93 & K91 & $\mathrm{U}$ & K13 & $\mathrm{T}$ & 0.001 & 69 & 6.9 \\
\hline MOCCA- 58 & $300 \mathrm{k}$ & 0.2 & $\mathrm{P}$ & K93 & K91 & $\mathrm{U}$ & UL & $\mathrm{TE}$ & 0.001 & 69 & 6.9 \\
\hline MOССA-59 & $300 \mathrm{k}$ & 0.2 & $\mathrm{P}$ & K93 & K91 & $\mathrm{U}$ & $\mathrm{L}$ & $\mathrm{TE}$ & 0.001 & 69 & 6.9 \\
\hline MOCCA- 60 & $300 \mathrm{k}$ & 0.2 & $\mathrm{P}$ & K93 & K91 & $\mathrm{U}$ & K95 & $\mathrm{TE}$ & 0.001 & 69 & 6.9 \\
\hline мосСA-61 & $300 \mathrm{k}$ & 0.2 & $\mathrm{P}$ & K93 & K91 & $\mathrm{U}$ & K95E & $\mathrm{TE}$ & 0.001 & 69 & 6.9 \\
\hline MOCCA- 62 & $300 \mathrm{k}$ & 0.2 & $\mathrm{P}$ & K93 & K91 & $\mathrm{U}$ & K13 & $\mathrm{TE}$ & 0.001 & 69 & 6.9 \\
\hline MOCCA-63 & $600 \mathrm{k}$ & 0.2 & $\mathrm{P}$ & K93 & K91 & $\mathrm{U}$ & K13 & $\mathrm{TE}$ & 0.001 & 55 & 5.5 \\
\hline
\end{tabular}

Table 2. For description see Tab. 1

parameters. Easy estimations indicate that a simulation for Big Survey will take 10-15 TBs. Thus, it is crucial to simplify this process and make the data analysis as straightforward as possible.

One of great advantages of the scripts developed for the моссA simulations is that they are ready for High Performance Computing. They can be executed on clusters of computers and analyze simulations in parallel if needed. In this way one can start series of jobs simultaneously for many simulations and get results much faster. The scripts have all of the dependencies built-in (they work on any machine equipped with Java). This feature is also very important for the future Big Survey project.

As a result of the extensive data analysis of the Mocca simulations many useful scripts were developed. A few of them are described here.

One of the most complicated scripts reads the data from the output of the MoccA simulations and prepares a detailed summary of properties of BSs. It checks dozens of parameters like masses at the time when a star has been recognized to be a BS and when it stopped to be the BS. It checks the time of the last mass transfer or merger (the event which actually creates a BS), to check whether a BS was created immediately or rather was dormant for some time. The script saves positions of BSs at the time of the detection and when it stops to be a BS. It checks if/when the BS escapes from GC, it stores information about initial channel of formation of each BS (see Sect. 3.2) and the changes of types due to e.g. dynamical interactions. The script stores also many other parameters. All of this provides a detail information on the history of formation and changes of properties of BSs.

Another example of a complex and time-saving script is the one which follows the complete history of a selected star. The script reads the whole output of the MoccA code and follows every possible event which concerns the selected star. It gathers all information on stars' properties (masses, radii, luminosity), all information on the dynamical interactions, stellar evolution events, etc. It follows also any change in the radial distance or in velocities available in the output. As a result, the script builds the complete history of the star, so one can study the evolution of masses, positions or binary properties. The script is very complicated since following the whole history of a star is not an easy task. Such star can change its identifier due to a merger event or it can change its binary companion. Thus, the properties of a given star may be stored in different columns in the same file. The script traces the history of the star starting from the end, it moves back in time and follows all these events as well as the history of the stars' predecessors (before mergers), until it reaches the first star at the time $T=0$. In this way one can study in detail the complete stellar and dynamical evolution of any star in the system.

The last example which shows the power of the scripts concerns gathering data from all available моссA simulations. The script traverse through all selected directories. It looks for моссA simulations and extracts some useful information from them. In this way one can study the properties of the whole set of GCs together. This script was extensively used e.g. to study the ratio between BSs in binaries and as single stars (see Sect. 3.3).

\section{PROPERTIES OF BLUE STRAGGLERS}

This section presents how initial conditions of GCs influence the population of BSs of different types. It is a continuation of the work published by Hypki \& Giersz (2013), where the channels of formation were presented and discussed in detail but only for a single test model.

The last section of this chapter presents in detail the ratio between BSs in binaries and as single stars. Some hidden properties of different populations of BSs might be revealed by this ratio.

Before the influence of various initial conditions on different populations of BSs will be discussed, the channels of formations have to be introduced. Then, rough estimates of the errors of numbers of blue stragglers will be presented.

\subsection{Channels of formation of BSs}

The first type of formation of BSs is called Evolutionary Merger (EM) and represents the scenario when two stars from a binary merge into one star. The merger is a result of the stellar evolution 
only, without involving other stars through dynamical interactions. The second channel is called Evolutionary Mass Transfer (EMT). This scenario creates BSs through a mass transfer in a binary, so that the mass of one of the stars overcomes the turn-off mass. In this case the stellar evolution does not have to lead immediately to a binary merger. A merger can occur later, and then, if the star would be the main-sequence star, it would still be considered as BSs. The third channel is called Evolutionary Dissolution (ED). It is the scenario when the stellar evolution leads to a disruption of a binary (e.g. SN explosion) with some mass accretion by the companion, which in consequence becomes a BSs. The EM, EMT and ED channels are connected to stellar evolution only.

Channels of formation of BSs which we include in the dynamical category are connected strictly to dynamical interactions and are described by the following cases. The channel of formation called Collision Single-Single (CSS) describes a physical collision between two single stars. This is the only channel, both from evolution and dynamical categories, which involves only two single stars. All other channels of formation involve at least one binary. The second channel called Collision Binary-Single/Binary (CBS, $\mathrm{CBB}$ ) describes the scenario when there is a collision between any two or more stars in a binary-single (CBS) or binary-binary interaction (CBB).

The rest of the channels do not in fact create a new BSs but rather describe the change of BSs type. Exchange BinarySingle/Binary, corresponds to the situation when BSs changes its companion in a binary, becomes a single star, or goes into a binary. EXBS stands for an exchange event in a binary-single dynamical interaction and EXBB means an exchange in a binarybinary interaction. The last dynamical channel is called Dissolution Binary-Single/Binary and corresponds to the scenario when BSs was present in a binary, which was disrupted by a binary-single dynamical interaction (DBS) or binary-binary interaction (DBB). The EXBS, EXBB, DBS and DBB cannot be the initial types of BSs. Initial BSs type can be EM, EMT, ED, CSS, CBS or CBB, and only later BSs can change its type into another one.

More details on the definitions of BSs and the physical processes of their creation one can find in Hypki \& Giersz (2013, Sect. 4.1).

The fluctuations of the number of BSs were discussed in Hypki \& Giersz (2013, Fig. 2, Fig. 3). The error $\sigma \sim 5$ BSs is a standard deviation of the mean number of BSs computed from five simulations with the same initial conditions but with different initial seed values. Thus, it is safe to assume that the fluctuations are \pm 10 BSs $(2 \sigma)$. Every feature, in terms of the number of BSs, which is of the order of 10 should not be considered.

\subsection{Influence of initial conditions on populations of BSs}

The initial conditions of simulations used in this section are summarized in Tab. 1 and Tab. 2. The simulations differ in the initial properties of semi-major axes, eccentricities, initial mass functions for single and binary stars, and different pairing of stars in binaries.

The changes of BSs populations are discussed only through one parameter at once to avoid any additional complexity. As a reference simulation we chose the MOccA-12 model (see Tab. 1). This model contains 300k initial stars, $20 \%$ of primordial binaries. Its initial tidal radius is $69 \mathrm{pc}$ and the half-mass radius is $6.9 \mathrm{pc}$. The MOcCA-12 model is called MOcCA-REF in this section for clarity.

\subsubsection{Properties of MOCCA-REF reference simulation}

The properties of the MOCCA-REF model are presented in Fig. 1. The meaning of the plots in this Figure, starting from the top-left corner, is the following. The first plot presents a few characteristic radii, like $r_{c}, r_{h}$ and $r_{\text {tid }}$. Please note, that the $r_{c}$ is slowly getting smaller. It will be important in the discussion on the influence of the initial concentration on the populations of BSs (Sect.3.2.4). The second plot shows the initial distribution of mass ratios for binaries (denoted as $U$ (uniform) in Tab. 1 and Tab. 2). The plot does not resemble uniform (flat) distribution because not all initially drawn mass ratios can be chosen - they cannot exceed the maximum mass for a binary $\left(100 M_{\odot}\right)$ and the minimum mass of a star $\left(0.08 M_{\odot}\right)$. The third plot presents the initial distribution of semi-major axes of binaries. It is a uniform distribution in logarithmic scale, between $4 R_{n}$ and $100 \mathrm{AU}\left(R_{n}\right.$ is a star with the smallest radii). It is denoted as UL in Tab. 1 and Tab. 2. Here again, the distribution is not entirely flat. There are some missing compact binaries in this distribution around $\left(\approx 0\left[\log \left(R / R_{\text {Sun }}\right)\right]\right)$ - the semi-major axis cannot be too small because it would create an immediate merger just in the first call of the stellar evolution. The forth plot shows the initial distribution of eccentricities (thermal distribution, denoted with $\mathrm{T}$ in Tab. 1 and Tab. 2, it is uniform in $e^{2}$ ). There are here some missing binaries with high eccentricities too. For some of the binaries eccentricity cannot be close to 1 because it would create an immediate merger too. The fifth plot shows the number of BSs of different types at a given time. BSs can change types (see Sect. 3.1), thus in the sixth plot there is the number of BSs of different initial types (the types of BSs at the time of their creation). Here the differences between the initial and present populations of BSs are not significant. They are more important for initially more concentrated clusters (see e.g. Sect. 3.2.4).

The number of EM BSs increases within the first few Gyr (see Fig. 1) as a result of two formation scenarios. EM in the first few Gyr are formed due to the Roche lobe overflow in compact binaries. The semi-major axis of the binary decreases slightly, after some time the heavier star leaves the main-sequence and its radius increases. The semi-detached phase starts, which leads to a merger. The second scenario of EM formation involves magnetic braking for slightly wider binaries and works for stars with masses less than about $1.25 M_{\odot}$. Around the time $3 \mathrm{Gyr}$, the turn-off mass equals $1.25 M_{\odot}$ and magnetic braking starts to work for both components in the binary (if they are main-sequence stars). This causes that the EM channel is most efficient around that time. The peak of EM channel for the MOCCA-REF model (and many others) is around $5 \mathrm{Gyr}$ (for more details see Hypki \& Giersz (2013, Sect. 4.1.3)).

The EMT channel is the most active in the model MOCCA-REF (and many others) during the first few Gyr as a result of the initial binary properties (see Fig. 1). There are two scenarios of forming EMT BSs. The first one creates EMT through the mass transfer in the Roche lobe overflow in a compact binary. In the second scenario in a wide binary a mass is transfered through stellar winds when a companion goes through the AGB phase. Both scenarios of formation of EMT BSs are the most active during the first few Gyr because the mass transfer concerns compact binaries and wide binaries together (Hypki \& Giersz 2013, Fig. 5). During the first few Gyr the mass transfer is possible for the largest number of binaries. The significance of EMT decreases with time because the mass transfer is less effective for less massive stars (for more details see Hypki \& Giersz (2013, Sect. 4.1.2)).

The number of the dynamical BSs (DBS, DBB) increases steady with time. It is caused by the increasing density in the GC 

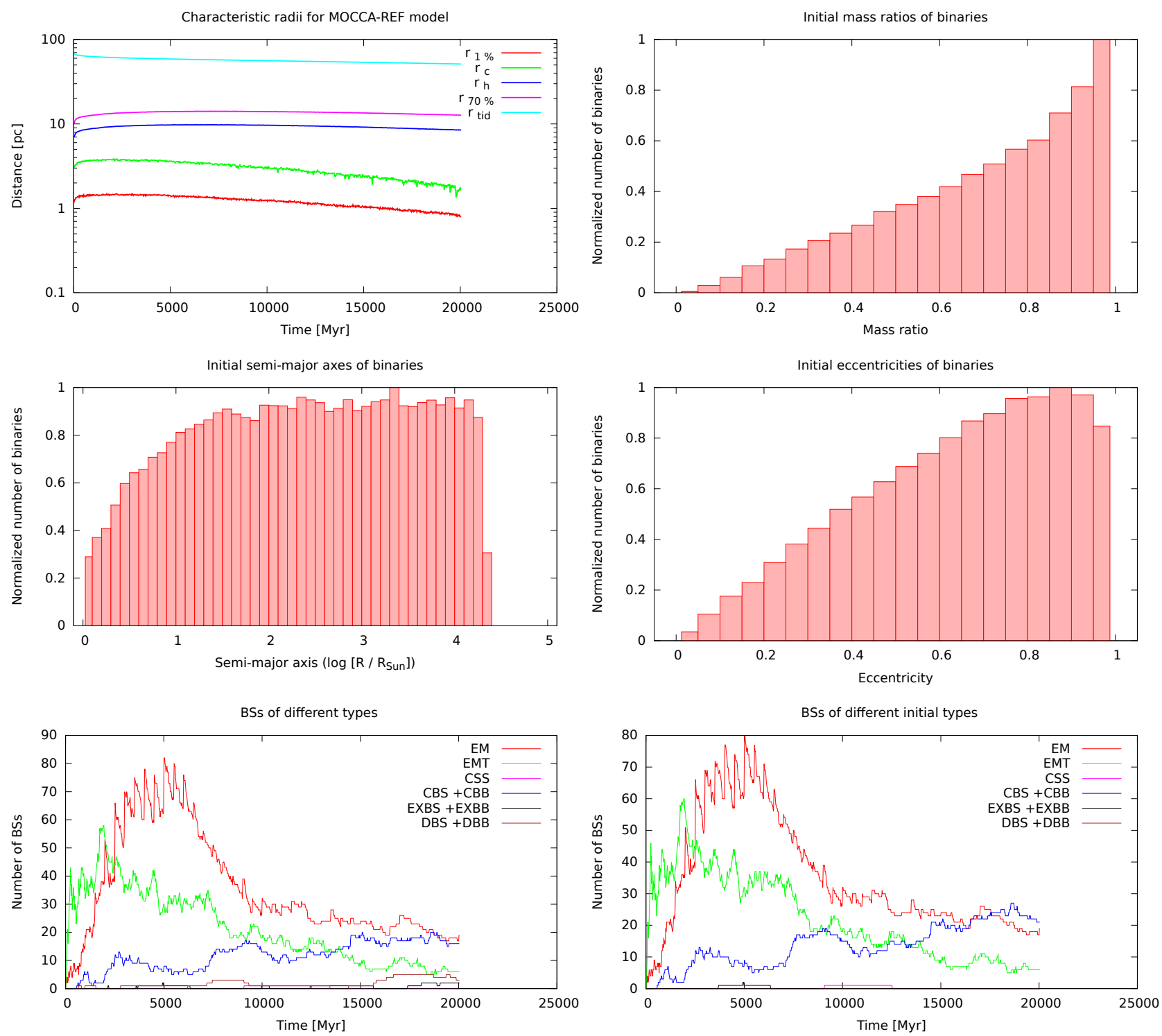

Figure 1. Properties of the MOCCA-REF model which is used in the Sect. 3.2 as a reference model to study how different initial conditions influence the population of BSs of different types. The first plot (top-left) presents several characteristic radii like $r_{c}, r_{h}$ and $r_{\text {tid }}$. The second plot (top-right) shows initial distribution of mass ratios for binaries (denoted as $\mathrm{U}$ in Tab. 1 and Tab. 2). The third plot (middle-left) shows the initial distribution of semi-major axes of binaries. It is a uniform distribution in logarithmic scale, between $4 R_{n}$ and $100 \mathrm{AU}\left(R_{n}\right.$ is a star with the smallest radii). It is denoted as UL in Tab. 1 and Tab. 2 . The next plot (middle-right) presents the initial distribution of eccentricities (thermal distribution - T in Tab. 1 and Tab. 2). The last two plots show the number of BSs of present types of BSs (bottom-left) and the initial types of BSs (bottom-right). See the text for a description.

(see radii in Fig. 1). Eventually, the dynamical BSs, at least for the MOCCA-REF model, become more important than EMT and more or less as numerous as from the EM channel.

MOCCA-12 is a very standard model which slowly evolves toward the core collapse. Its density in the core raises during the Hubble time so that the number of BSs created due to dynamical interactions becomes important. It is a very reasonable standard model of a real size GC. The only significant difference as compared to real clusters is a slightly larger fraction of primordial binaries. Usually, in GCs one can observe a fraction $\lesssim 10 \%$, whereas the мосcA12 has $20 \%$. The larger number is chosen to have a larger number of BSs and thus to highlight their features. The chosen $r_{h}$ is also slightly larger than for a typical GC. Such value of $r_{h}$ was chosen to give us a freedom in both increasing and decreasing its value for other models.

\subsubsection{Influence of semi-major axes distribution on BSs population}

This subsection shows how different semi-major axes distributions change the populations of BSs.

Fig. 2 shows the differences between the reference model MOCCA-REF and the model MODEL-54. The properties of the MOCCAREF were discussed in the Sect. 3.2.1. The difference between models concerns only the initial semi-major axes distributions (top plot in Fig. 2). The reference model MOcCA-REF has a uniform distribution in $\log$ scale $(\mathrm{a}=\mathrm{UL}$, red line, top plot $)$ and the model моссA-54 
Semi-major axes distribution for MOCCA-REF and MOCCA-54 simulations

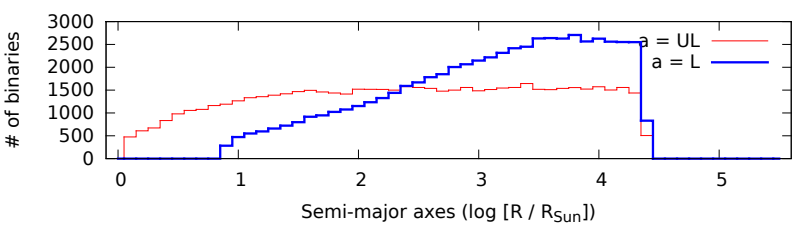

Actual type of BSS

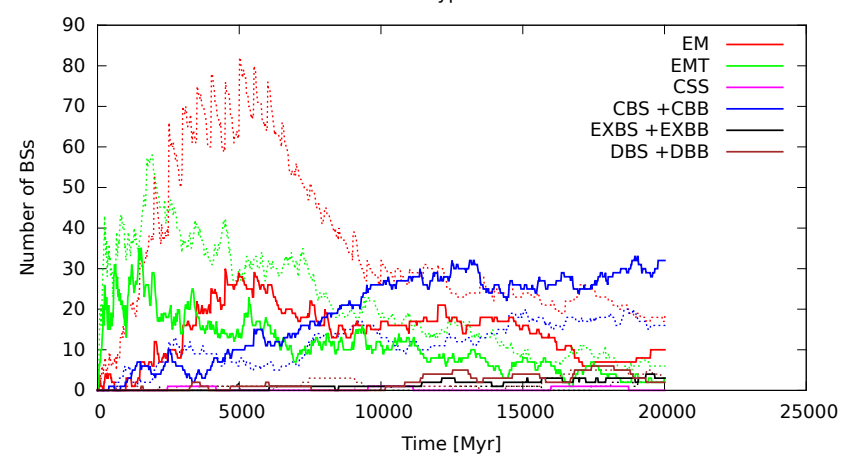

Figure 2. Comparison between the reference model MOCCA-REF and the model MOccA-54. The difference between models concerns the initial semimajor axes distributions (top). The reference model MOCCA-REF has a uniform distribution in log scale ( $\mathrm{a}=\mathrm{UL}$, red line, top plot), while the model MOcCA-54 has lognormal distribution ( $\mathrm{a}=\mathrm{L}$, blue line, top plot). The result differences in the number of BSs of different types is presented in the bottom plot. BSs from the MOCCA-REF model are presented with dashed lines, whereas the BSs from Mocca-54 models with solid lines. For definitions of BSs types see Sect. 3.1 and for details see the text.

has lognormal distribution $(\mathrm{a}=\mathrm{L}$, blue line, top plot). The model MOCCA-REF has many more binaries with small and medium semimajor axes $\left(<10^{2.5}\left[R_{\odot}\right]\right)$. In turn, MoccA-54 has more binaries with longer semi-major axes $\left(>10^{2.5}\left[R_{\odot}\right]\right)$.

The differences in semi-major axes distributions between MOCCA-REF and MOCCA-54 models yield significant differences in the channels of formation of BSs. The number of EMT and EM is significantly lower for MOсCA-54 model. However, the number of dynamical BSs (CBS, CBB) is higher. The number of exchanges and dissolution in BSs is not important for both models.

The lower number of EM and EMT BSs for Mocca-54 is a consequence of its semi-major axes distribution for which there is less compact binaries. Small semi-major axes are expected for EM BSs, because in order to have an evolutionary merger, one has to have a compact binary. Additionally, binaries in MOcCA-54 model need more time to have evolutionary mergers, thus, the number of EM BSs becomes significant after $5 \mathrm{Gyr}$ - the number of EM for MOCCA-REF model is then already at its peak and is a dominant channel of formation. For more details about the physical processes of formation of EM see Hypki \& Giersz (2013, Sect. 4.1.3).

A similar explanation applies also for the lower number of EMT BSs for Mocca-54 model. There are two subgroups of EMT BSs. The first group consists of harder binaries ( $<10$ days) for which there is some Roche lobe overflow. The second group consists of wider binaries (> 100 days) for which future BS gains some additional mass trough stellar winds, when the companion goes through the AGB phase. In the second subgroup the eccentricities are significantly larger then 0.1 (even 0.9 ) which makes the mass transfer a bit easier. The second subgroup creates less BSs than the first one. In мосса-54 there is much less compact binaries (see top panel in Fig. 2) thus the number of EMT is smaller too.
Semi-major axes distribution for MOCCA-REF and MOCCA-55 simulations
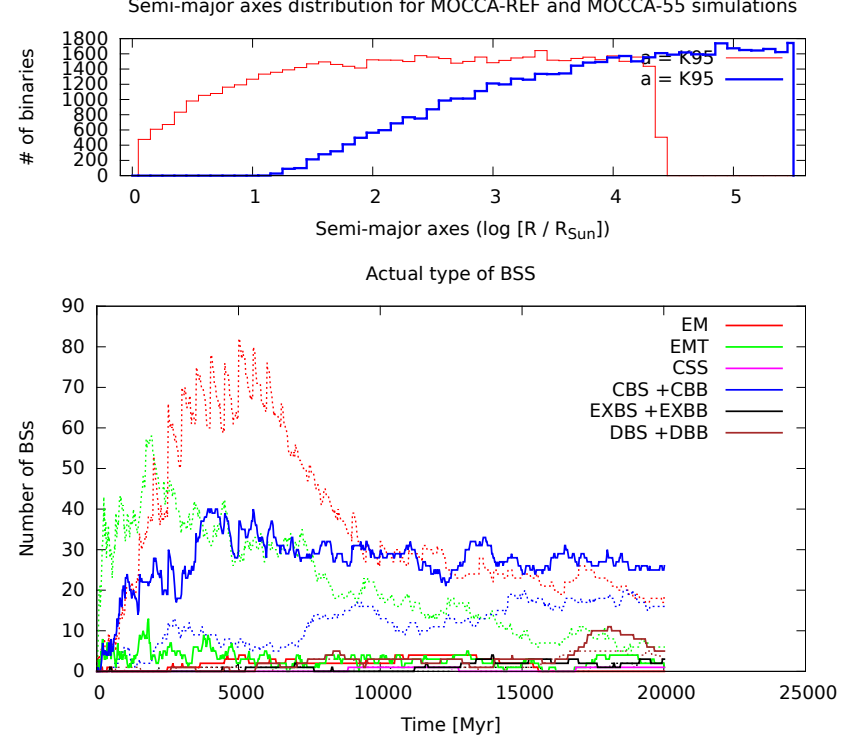

Figure 3. Comparison between the reference model MOCCA-REF and model MOcca-55. The difference between models concerns the initial semi-major axes distributions (top). The reference model MOCCA-REF has a uniform distribution in log scale $(\mathrm{a}=\mathrm{UL}$, red line, top plot) and the model MoccA-55 has the distribution from Kroupa (1995b) $(\mathrm{a}=\mathrm{K} 95$, blue line, top plot). The result differences in the number of BSs of different types are presented in the bottom plot. BSs from the MOCCA-REF model are presented with dashed lines, whereas the BSs from MoccA-55 models with solid lines. For definitions of BSs types see Sect. 3.1 and for details see the text.

For detail description of the physical processes of formation of both subgroups of EMT channel see Hypki \& Giersz (2013, Sect. 4.1.2).

Less intuitive explanation concerns the higher number of dynamical BSs (CBS, CBB) for Mocca-54 model. The dynamical BS is created due to a physical collision (or collisions) which occurs during a dynamical interaction. For моссA-54 there are in overall many more dynamical interactions because it contains a larger number of wider binaries (see top panel in Fig. 2). There are $35 \mathrm{k}$ dynamical interactions for MOCCA-REF model within $20 \mathrm{Gyr}$ and 60k for Mocca-54. It is almost twice as many. As a result, there is also more physical collisions (140) during these interactions for моссA54 model, whereas for MOcCA-REF there are only 80 collisions. The models are identical, except the semi-major axes distributions. The number of binaries and the concentration are the same, the GCs for both models evolve very similarly, the characteristic radii like $r_{c}$, or $r_{h}$ are very similar too.

The only difference between MOCCA-REF and MOCCA-54 is that for MoсcA-54 the average semi-major axes for binaries are larger. Wider binaries have larger probabilities of having dynamical interactions. Many of them are in fact only distant fly-by interactions, which do not change significantly semi-major axes. However, these interactions increase the eccentricities. Larger eccentricities increase the probabilities of the collisions further. At some point, the FEWBODy code detects a collision, when the periastron distance gets smaller than the sum of the radii of stars. As a result there are more dynamical BSs (CBS and CBB) for the models which have initial semi-major axes distribution containing more wide binaries, despite the fact that the initial concentrations for both models are the same (e.g. Mocca-54). This scenario of formation of the BSs is discussed in details in Sect. 3.3.7.

Fig. 3 shows the differences between the reference model MOCCA-REF and another model, MODEL-55. The difference concerns 
only the initial semi-major axes distributions (top plot in Fig. 3). The reference model MOcCA-REF has a uniform distribution in log scale and the model Mocca-55 has the distribution of Kroupa (1995b), which results in the given semi-major axes distribution (a $=\mathrm{K} 95$, blue line, top plot). The model MOcCA-REF has many more binaries with small and medium semi-major axes $\left(<10^{2.5}\left[R_{\odot}\right]\right)$. In turn, MoccA-55 has binaries with larger semi-major axes $(>$ $\left.10^{4.5}\left[R_{\odot}\right]\right)$. Fig. 3 shows that the number of EMT, EM is very low for MOсCA-55 model. However, the number of dynamical BSs (CBS, CBB) is much higher.

When it comes to the differences between MOcCA-55 and previously discussed model moсcA-54 (see Fig. 2), the мосса-55 has even less binaries with semi-major axes $<10^{2.5}\left[R_{\odot}\right]$ than model MOCCA-54. This has an influence on the number of BSs. Previous MOCCA-54 model has the average number of EM around 20 and EMT around 15. Whereas the Mocca-55 models has only a few of EM and EMT BSs. The number of CBS and CBB BSs increase faster for мосса-55 model until reaching the average value around 30 - the same as for the previous MoccA-54 model.

The differences in the number of BSs for the MOcCA-REF and MOCCA-55 models confirm and make it even more explicit to notice the previously stated conclusions. The EM and most of EMT BSs are created from compact binaries. Model MoccA-55 has the lowest number of such binaries and thus the number of BSs of these types is not significant at all. There are on average only a few EM and EMT BSs for model MOcсA-55.

The number of CBS and CBB BSs is larger for model MoccA55 than for MOCCA-REF and previously discussed model MOcCA-54. The explanation is the same as previously. For mocca-55 model there are more binaries with larger semi-major axes. Thus, the probability of dynamical interactions is higher. The interactions increase eccentricities to such values that binaries eventually merge. For MOCCA-REF model for the first 5 Gyr there were around $17 \mathrm{k}$ dynamical interactions, whereas for the MOcCA-55 there were already $176 \mathrm{k}$ interactions. Thus, the number of CBS+CBB BSs increases so fast during the first $5 \mathrm{Gyr}$. This is also the reason why the number of CBS and CBB BSs in MOcCA-55 increases faster than for MoccA-54 model. There are many more dynamical interactions which lead to collisional events. Interestingly, the number of $\mathrm{CBS}+\mathrm{CBB}$ raises to the same level of about $30 \mathrm{BSs}$ after the first $5 \mathrm{Gyr}$ for both models. The reason why the number of CBS and CBB stopped to increase for MOссA-55 model is caused by the number of binaries destroyed by dynamical interactions. The number of destroyed binaries for MOCCA- 55 after $10 \mathrm{Gyr}$ is $28.3 \mathrm{k}$ binaries, whereas for MOcCA- 54 it is only $3.5 \mathrm{k}$. Most of the widest binaries were destroyed and thus the number of CBS+CBB stopped to increase.

The last remark is needed to complete the discussion on the influence of the initial semi-major axes distribution on the populations of BSs. The mocca code allows to change the maximum semi-major axis for binaries. By default it is $100 \mathrm{AU}\left(\sim 10^{4.3}\left[R_{\odot}\right]\right)$. Of course, if one set this value to a larger one, the number of BSs of different channel would change. However, it is expected to see differences obeying the conclusions specified in the previous paragraphs. It is expected to see more EM and EMT BSs if there are initially more compact binaries. And when the number of wide binaries is larger, then the number of dynamical BSs should increase. The maximum values of semi-major axes for the MOCCA-REF and MOcCA-54 models are the same (100 AU). Only mocca-55, which generates semi-major axes according to Kroupa (1995b), sets up its own value for maximum semi-major axis. Testing the influence of wider orbits than $100 \mathrm{AU}$ on the population of BSs is planned for the future research.
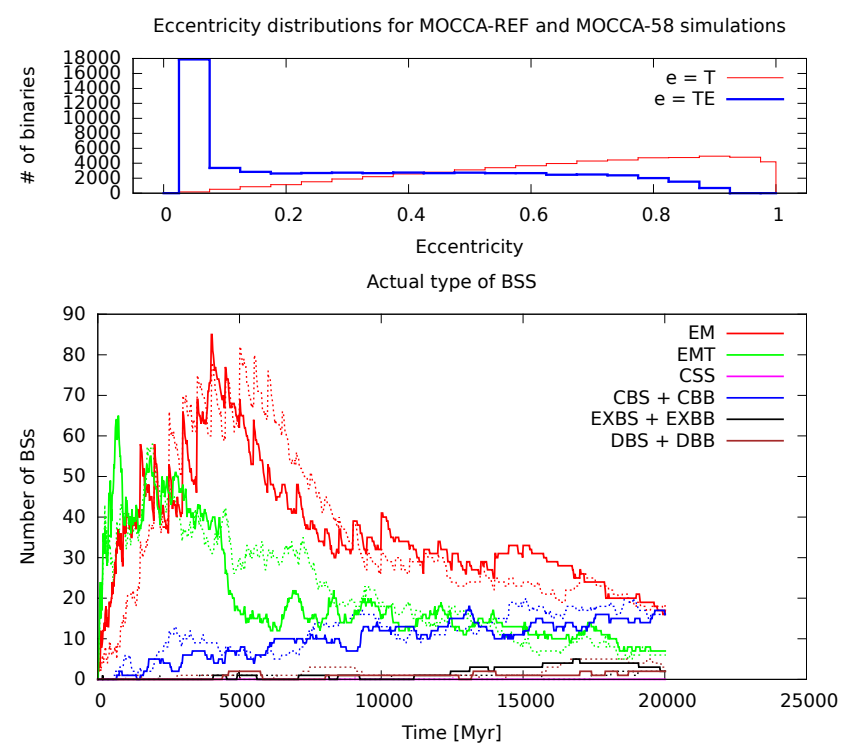

Figure 4. Comparison between the reference model MOCCA-REF and model MOCCA-58. The difference between models concerns the initial eccentricity distributions (top). The reference model MOCCA-REF has a thermal distribution ( $\mathrm{e}=\mathrm{T}$, red line, top plot) and the model MoccA-58 has a thermal distribution with eigenevolution Kroupa (1995b) (e = TE, blue line, top plot). The result differences in the number of BSs of different types are presented in the bottom plot. BSs from the MOCCA-REF model are presented with dashed lines, whereas the BSs from Mocca-58 model with solid lines. For definitions of BSs types see Sect. 3.1 and for details see the text.

\subsubsection{Influence of eccentricity distribution on BSs population}

Fig. 4 shows how different initial eccentricity distributions (top panel) influence the population of BSs of different types (bottom panel). The model MoccA-REF has a thermal distribution $(\mathrm{e}=\mathrm{T})$ and the model MоссA-58 has a thermal distribution as well but with a procedure called eigenevolution applied to the eccentricity distribution (not applied to orbital periods) (Kroupa 1995b). The basic idea behind eigenevolution is to modify a binary for small pericenter distances due to pre-main-sequence evolution, when stellar radii are larger. In this procedure eccentricity is sampled from a thermal distribution. A pre-main-sequence radius of a star of $5 \mathrm{~m}^{1 / 2}\left[M_{\odot}\right]$ is used to reflect the earlier contraction stage. Using the tidal circularization theory (Mardling \& Aarseth 2001), modified eccentricity $e_{i}$ for characteristic time interval of $10^{5} \mathrm{yr}$ is computed. Angular momentum conservation is used afterwards to compute semi-major axes. Finally, any case of overlapping enlarged radii is defined as collision. It is rejected and the procedure is repeated. As a result of the eigenevolution procedure some fraction of compact binaries are circularized (see top plot in Fig. 4).

Fig. 4 shows that the population of evolutionary BSs is not affected significantly by the changed eccentricity distribution. The overall number of EM and EMT BSs is very similar for both models. The only noticeable difference is that the EM channel raise a bit faster for мосса-58 model. The eigenevolution procedure circularize some of the compact binaries, thus the EM channel seems to be raising in the beginning of the simulations more quickly EM BSs do not need any additional time to circularize their orbits and finally merge. However, the changes are within the fluctuations ( \pm 10 BSs) thus cannot be recognised as certain.

The population of dynamical BSs is not affected by the different eccentricity distribution. The eccentricities neither have in- 


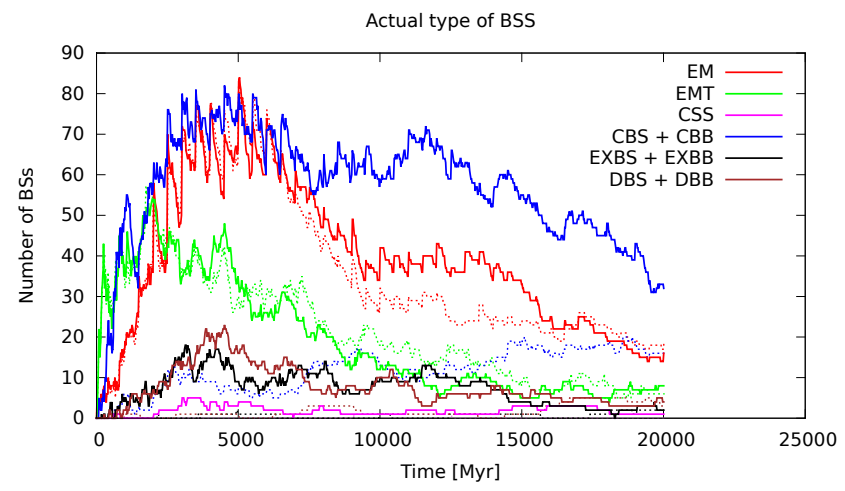

Figure 5. Comparison of the number of BSs of different types between the reference model MOcCA-REF (dashed lines) and model MoccA-8 (solid lines). The difference between models concerns only the initial concentration $c=$ $r_{t i d} / r_{h}$. For the MOCCA-REF $c=10$, and for the MOCCA- $8 c=30$. The reference model MOCCA-REF is described in details in Sect. 3.2. For definitions of BSs types see Sect. 3.1 and for details see Sect. 3.2.4.

fluence on the probabilities of the dynamical interactions, nor the probabilities of the collisions. Thus, the populations of CBS and CBB for both models are well within the fluctuations.

\subsubsection{Influence of concentration on BSs population}

Fig. 5 shows how the population of BSs of different types depends on the cluster concentration parameter. It shows differences between MOcCA-REF model with concentration $c=r_{\text {tid }} / r_{h}=10$, and MoccA- 8 model with $c=30$. All other parameters between these models are the same.

The number of evolutionary BSs (EM, EMT) is the same for both models. There are some small differences but they fall well within the error $( \pm 10 B S s$, see Sect. 3.1). The same number of EM and EMT BSs for the models suggests that the concentration does not influence their population. Much more important for them are the initial conditions - especially the semi-major axes (see Sect. 3.2.2). This suggest also that the evolutionary BSs have been mostly created in unperturbed, primordial binaries. The binaries, which later on created BSs, were not affected by close interactions, even for the MоссA- 8 model which has much greater concentration.

The differences between MOcCA-REF and MOcCA- 8 models concerns the number of dynamical BSs (CBS, CBB). For the more concentrated model, MOсCA- 8 , their number increases fast and this channel of formation becomes dominant just after $\sim 7$ Gyr. GCs with higher concentrations have higher probabilities of interactions. Thus, the number of physical collisions also increases. Interestingly, for higher concentrations the number of BSs of types EXBS, EXBB, DBS, and DBB increases too (black and blue lines in Fig. 3.2.4). It is caused by the numerous, strong dynamical interactions in which BSs change their companions or are dissolved. It is the natural outcome of the highly concentrated systems.

Even the larger concentration, $c=40$ (MосcA-7, see Tab. 1), does not change the population of EM and EMT BSs. Their number is very much the same as for the model MocCA-REF (see Fig. 6). The number of the dynamical BSs is naturally larger. There are many more strong dynamical interactions leading to collisions. There are more exchanges and dissolutions of binaries as well (EXBS, EXBB, DBS, DBB).

The higher concentration $(c=40)$ puts even more confidence into the fact that EM and EMT BSs seem to be formed in primor-

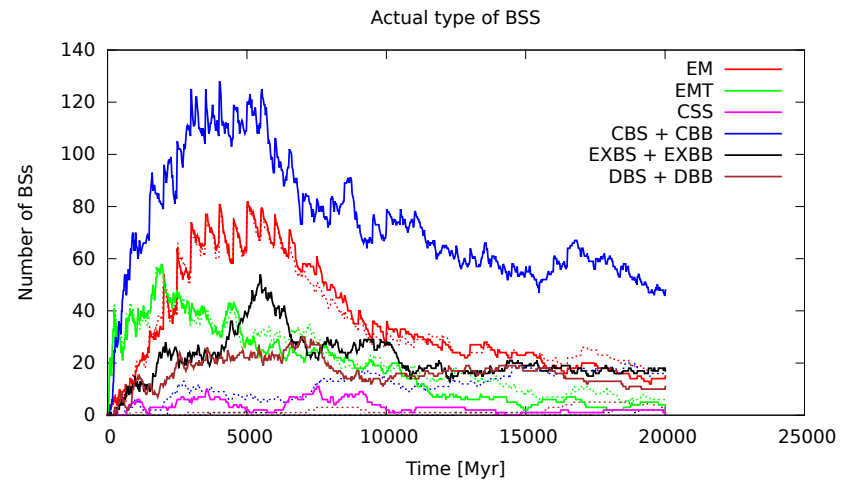

Figure 6. Comparison of the number of BSs of different types between the reference model MOCCA-REF (dashed lines) and model MoccA-7 (solid lines). The difference concerns only the initial concentration $c=r_{t i d} / r_{h}$. For the MOCCA-REF $c=10$, and for the MOCCA-7 $c=40$. The reference model MOCCAREF is described in details in Sect. 3.2. For definitions of BSs types see Sect. 3.1 and for details see Sect. 3.2.4.

dial, unperturbed binaries. In Fig. $5(c=30)$ there is some difference in the number of EM between $\sim 10 \mathrm{Gyr}$ and $\sim 15 \mathrm{Gyr}$. However, these are most likely only some fluctuations, because for the higher concentration there is no such discrepancy ( $c=40$, Fig. 6).

\subsubsection{Relation between hard and soft binaries}

Different initial semi-major axes distributions and initial clusters' concentrations have variuos influence on the populations of dynamical BSs because of (i) the boundary between hard and soft binaries and (ii) the probabilities of the dynamical interactions. The boundary between hard and soft binaries plays a very important role in GCs. Hard binaries have binding energies larger than the average kinetic energies of stars, whereas soft binaries inversely. Heggie (1975) showed that in general hard binaries, due to dynamical evolution of GCs, get harder, and soft binaries get softer (wider) or disrupt.

The division between the hard and soft binaries can be expressed as an average binary semi-major axis of a GC.

The cluster binding energy is equal to $E_{b}=-\frac{0.4 G M^{2}}{r_{h}}$, where $\mathrm{G}$ is the gravitational constant, $\mathrm{M}$ - the total mass, and $r_{h}$ - the half-mass radius. The cluster kinetic energy equals $E_{k}=0.5 M v_{h}^{2}$, where $v_{h}$ is the average velocity in the system. Thus, using the virial theorem, the average velocity can be expressed as $v_{h}^{2}=\frac{0.8 G M}{r_{h}}$. The total mass can be expressed as $M=\bar{m} N$, where $\bar{m}$ is the average mass in the system and $\mathrm{N}$ - the number of stars.

The boundary semi-major axis $(\bar{a})$ between soft and hard binaries can be derived from the equality between average kinetic and binding energy of a binary:

$0.5 \bar{m} v_{h}^{2}=\frac{G \bar{m}^{2}}{2 \bar{a}} \Rightarrow \bar{a}=\frac{G \bar{m}^{2}}{\bar{m} v_{h}^{2}}$

using expressions for $v_{h}^{2}$, and $\mathrm{M}$ we get:

$\bar{a}=\frac{G M r_{h}}{N 0.8 G M}=\frac{r_{h}}{0.8 N}$

In a single time step, a probability of a strong dynamical inter- 
action between a binary and another object (single star or binary) is given by:

\section{$P=\sigma_{h} n_{h} u_{h} \Delta t$}

where $\sigma_{h}=\pi \bar{a}^{2}\left(\frac{2 G\left(m_{b}+\bar{m}\right)}{\bar{a} u_{h}}+1\right)$ is the cross section for the interaction, $u_{h}$ - the relative velocity at the infinity, $n_{h}$ - the local number density of stars (single or binaries), $m_{b}$ - the average mass of a binary, and $\Delta t$ is the time step. It is assumed that the strong interaction occurs when an incoming object approaches binary at the distance comparable to the binary semi-major axis. The relative velocity can be expressed by the average velocity as $u_{h}^{2}=2 v_{h}^{2}$, the average mass of the binary as $m_{b}=2 \bar{m}$, and taking the expression for $v_{h}^{2}$, we get:

$\sigma_{h}=\pi \bar{a}^{2}\left(\frac{2 G 3 \bar{m}}{\bar{a} 2 v_{h}^{2}}+1\right)=\sigma_{h}=\pi \bar{a}^{2}\left(\frac{3}{\bar{a}} \frac{r_{h} \bar{m}}{0.8 M}+1\right)=\pi \bar{a}^{2}\left(\frac{3}{\bar{a}} \frac{r_{h}}{0.8 N}+1\right)$

using expression for $\bar{a}$, we get:

$\sigma_{h}=4 \pi \bar{a}^{2}$

The local number density (computed only inside $r_{h}$, thus only half of the mass is taken) is:

$\rho_{h}=\bar{m} n_{h}=\frac{3 M}{8 \pi r_{h}^{3}} \Rightarrow n_{h}=\frac{3 N}{8 \pi r_{h}^{3}}$

Using the above expressions and simplifying the probability for the strong dynamical interactions, we get:

$P=\frac{1.5 \sqrt{1.6 G}}{0.8^{2}} \frac{M^{1 / 2}}{N r_{h}^{2}} \propto \frac{\bar{m}}{M^{1 / 2} r_{h}^{3 / 2}}=\frac{\bar{m}^{1 / 2}}{N^{1 / 2} r_{h}^{3 / 2}}$

From the Eq. 1 we have $\bar{a} \propto \frac{r_{h}}{N}$ and from the Eq. 5, $P \propto \frac{1}{N^{1 / 2} r_{h}^{3 / 2}}$. The influence of the initial semi-major axes distributions and initial concentrations of the GCs on the CBS and CBB BSs is a result of the interplay between these two equations.

In the case of different semi-major axes distributions (but the same $r_{h}$ and $\mathrm{N}$ ) the average semi-major axis $\bar{a}=1275\left[R_{\odot}\right]=$ $3.1\left[\log R_{\odot}\right]$ is the same for models MOcCA-REF, MOcCA-54 (see Fig. 2), and MoccA-55 (see Fig. 3). However, the boundary between hard and soft binaries $(\bar{a})$ causes that for MOсCA-54 there are more binaries which are softer (wider) from the point of view of the GC. There are even more softer binaries for the Mocca- 55 model. It causes that there will be many more dynamical interactions for the models with a large number of soft binaries. Mostly they are just fly-bys which continuously increases binaries' eccentricities. Eventually, because of their high eccentricities, more binaries collide. Thus, for the semi-major axes distributions with a larger number of soft binaries, the number of dynamical BSs is higher as well.

In the case of different initial clusters' concentrations (see Fig. 5 and Fig. 6), but the same initial semi-major axes distributions, the values of $\bar{a}$ decrease for the higher concentrations. For the MOCCA-REF model ( $r_{h}=6.9, c=10$, see Fig. 1$)$ the average semi-major axis is $\bar{a}=3.1\left[\log R_{\odot}\right]$, whereas for the model moccA-7 $\left(r_{h}=1.7, c=40\right)$ it decreases to $\bar{a}=2.5\left[\log R_{\odot}\right]$. Because of Eq. 5 the probabilities of dynamical interactions increase for higher concentrations (lower $r_{h}$, but the same N). For the MOCCA-REF model $P \propto \frac{1}{r_{h}^{3 / 2}} \propto 0.05$. In turn, for the MoccA-7 model $P \propto \frac{1}{r_{h}^{3 / 2}} \propto 0.45$ - the probability is 9 times higher. For higher concentrated models (smaller $\bar{a}$ ) there are more binaries with small semi-major axes which simultaneously are soft from the point of view of the GC. This causes that there are more collisions for them and the number of CBS and CBB increase with the higher concentrations.

For higher initial concentrations (smaller $\bar{a}$ ) the number of EM and EMT stays the same because binaries which created these BSs are hard for all models (MOcсA-REF, MOcсA-7, and Mocca-8). The value of $\bar{a}$ is not small enough to start to disrupt the binaries which lead to the creation of the EM and EMT BSs. Only the population of EMT which is created from wide binaries (through the stellar winds, see Sect. 3.1) could be affected. However, their number is low.

\subsection{Blue stragglers in binaries}

The ratio of the number of BSs in binaries and as single stars is a very interesting subject to study. It might reveal some hidden properties of BSs and lead to some methods of narrowing down the initial distributions of semi-major axes of binaries. This section presents an analysis of these ratios for all of the MосcA simulations described in Sect. 2.1.

Fig. 7 shows the ratios between BSs in binaries to BSs as single stars $\left(R_{B / S}\right)$ for the MoccA simulations (Y axis). The time on $\mathrm{X}$ axis is given in the units of the present half-mass relaxation times $\left(t_{r h}\right)$, but only up to 12 Gyr. It shows the dynamical scales of the GCs. The higher values are characteristic for dynamically older GCs. By using such a scale one can easily compare GCs with very different initial conditions. Each circle represents one ratio $R_{B / S}$ computed for one mocca simulation for one snapshot in time. The BSs from all channels are combined together into one circle. For each mocca simulation the ratio $R_{B / S}$ is computed every $1 \mathrm{Gyr}$ (thus, $\max 12$ circles for one simulation $-1 \ldots 12 \mathrm{Gyr}$ ). The sizes of circles decrease with time. For several selected simulations there are plotted lines to show overall evolution of the ratio $R_{B / S}$.

The lines with ratios $R_{B / S}$ in Fig. 7 for all мосcA simulations reveal two separate groups of models.

The first group of models, called MOCCA-DropPING, starts with the ratio $R_{B / S} \gtrsim 1.0$ (at $T=1 \mathrm{Gyr}$ ). Then, as the evolution of GCs proceeds, the ratio drops continuously until it settles around $R_{B / S} \sim$ 0.3 . This trend is well represented by models e.g. MoccA-39 (blue), моссA-33 (magenta), MоссA-34 (dark green). For a few models of this group there are observed some exceptions. The models mocca5 (red), MOcCA-12 (violet) raise after several Gyr to values around $R_{B / S} \sim 0.5$. For a few other models, e.g. мосса-32, моссA-24, the ratio drops to values $R_{B / S} \sim 0.2$ and intersects with models of the second group.

The second group of models, called Mocca-RAISING, starts with the ratios $R_{B / S} \lesssim 0.3$. The ratios stay at the same level or raise slightly with time (e.g. MOccA-63, light green; MOccA-55, yellow). The ratios from this group, in general, do not increase significantly above the level $R_{B / S} \sim 0.3$.

The models MOCCA-DROPPING and MOCCA-RAISING have various initial conditions (see Sect. 2.1). There are models which evolve slowly almost as isolated clusters, the other ones are very dense and thus they evolve quickly. Some models have a large number of compact binaries, whereas other have many wide binaries. Despite all these differences, all models seem to evolve towards the values $R_{B / S} \sim 0.3$. The level $R_{B / S} \sim 0.3$ seem to be a universal one.

The ratio $R_{B / S}$ computed for all channels together adds complexity. Thus, we decided to split the ratio for the evolutionary and dynamical BSs. Fig. 8 presents the ratio $R_{B / S}$ divided into evolutionary (top panel, $R_{B / S}^{\text {evol }}$ ) and dynamical BSs (bottom panel, $R_{B / S}^{d y n}$ ). This plot will be helpful in the discussion on the ratios $R_{B / S}$ in the 


\section{$\mathrm{R}_{\mathrm{B} / \mathrm{S}}$ ratio for all MOCCA simulations}

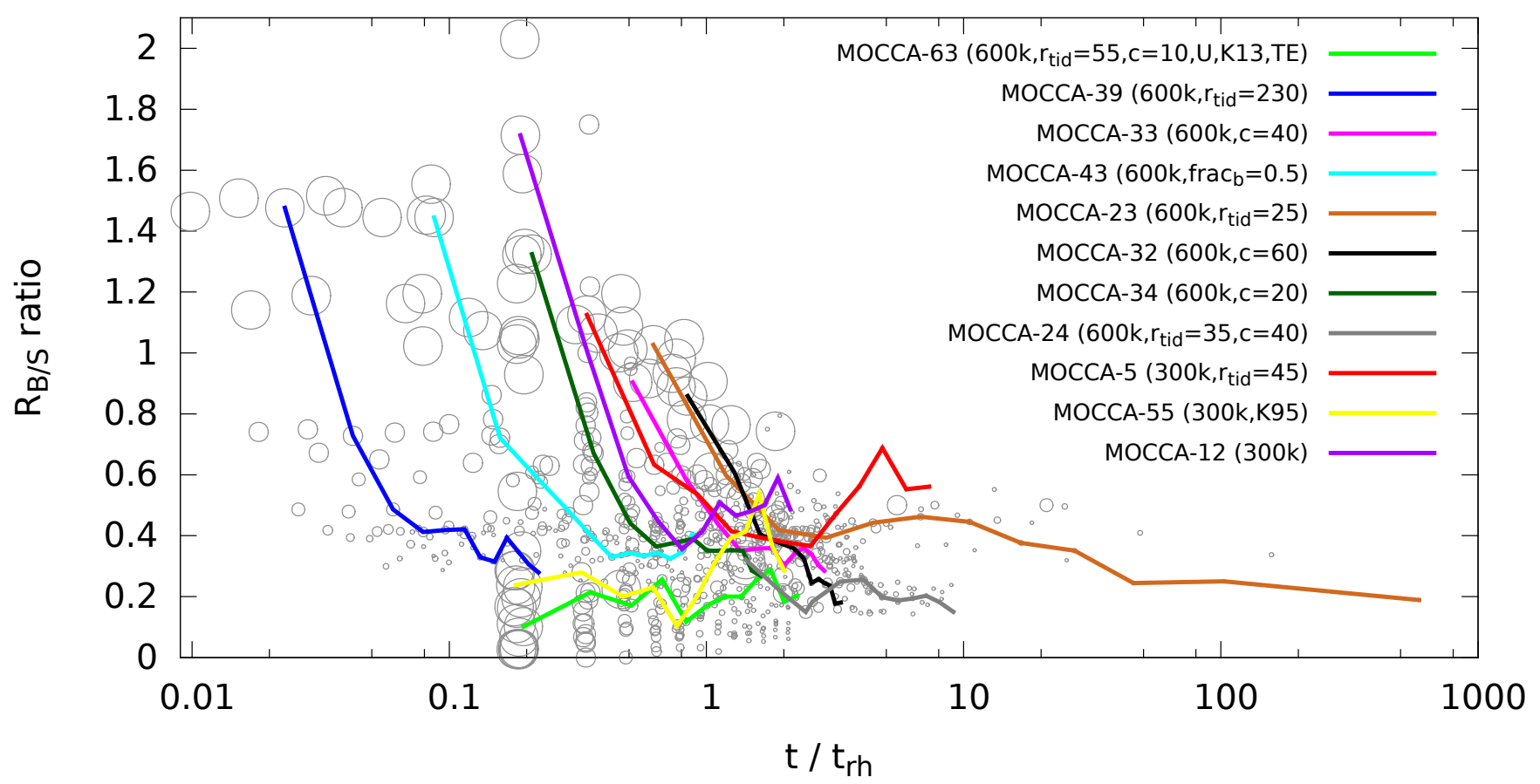

Figure 7. Evolving ratios between BSs in binaries and as single stars $\left(R_{B / S}\right)$ for all mocca simulations (Tab. 1, Tab. 2) as a function of time in the units of the present half-mass relaxation times $\left(t_{r h}\right)$ but only up to 12 Gyr. The X axis shows the dynamical scales of the GCs - higher values for dynamically older GCs. Each circle represents one ratio $R_{B / S}$ for one moccA simulation for one snapshot in time. The BSs from all channels are combined together into one circle. For each mocca simulation the ratio $R_{B / S}$ is computed every 1 Gyr (thus, max 12 circles for one simulation - 1...12 Gyr). The sizes of circles decrease with time. For several selected simulations there are plotted lines to show the evolution of the ratio $R_{B / S}$. The values in brackets are copied from Tab. 1 and Tab. 2 for clarity. They specify only these parameters which are different from the parameters of models moccA-35 and MoccA-12 (standard models for $600 \mathrm{k}$ and $300 \mathrm{k}$ respectively). For details see the text.

next paragraphs. In Hypki \& Giersz (2013, Sect. 4.1.5) one can find detail analysis of the changes of types of BSs for a typical simulation. In general, the evolutionary BSs do not often change their types. Additionally, Sect. 3.2 shows that the population of evolutionary BSs strongly depends on the initial distribution of semimajor axes. In turn, the concentrations of the GCs influence the population of the dynamical BSs. They change types if the density of GC is high enough. Thus, it is safe to assume that EM are single stars, EMT are in binaries, and CBS, CBB, EXBS, EXBB, DBS, DBB channels can be both in binaries as well as single stars. This allows to split $R_{B / S}$ without worrying about the changes of types of BSs.

\subsubsection{Models from MOCCA-DROPPING group}

Evolution of the ratio $R_{B / S}$ for the models from MOCCA-DROPPING group is mainly a consequence of the initial distribution of semimajor axes of binaries.

\subsubsection{MоссA-39}

A good representative of the MOCCA-DROPPING group is the MOcCA-39 model. It starts with $600 \mathrm{k}$ stars, $20 \%$ of primordial binaries, a large tidal radius $r_{\text {tid }}=230$, and small concentration $c=r_{\text {tid }} / r_{h}=10$ (see Tab. 1). Because of the large $r_{t i d}$, this model evolves almost as an isolated GC. The number of BSs of different types for this model are shown in Fig. 9 (top-left panel). The number of EM, and EMT is a pure consequence of the initial conditions (see Sect. 3.2.2). Because of the large $r_{\text {tid }}$ the number of BSs created due to dynamical interactions is negligible.

The ratio $R_{B / S}$ for model MoccA-39 is represented as blue line in Fig. 7. It has only EM and EMT BSs, and there is no significant contribution from dynamical BSs. The initial ratio $R_{B / S}$ is around 1.4 for time $1 \mathrm{Gyr}$ (the first circle in Fig. 7) because during that time the most active channel is EMT (see Fig. 9). After 4 Gyr the EM channel becomes the most dominant one. Then, the ratio drops to $R_{B / S} \sim 0.4$ and stays around this level up to $12 \mathrm{Gyr}$. The ratio does not change significantly during that period because the number of EM and EMT decreases at almost the same rate (see Fig. 9).

The EMT channel is the most active in the model Mocca-39 (and many others) during the first few Gyr as a result of the initial binary properties (see Sect. 3.1). The number of EM BSs increases within the first few Gyr as a result of two formation scenarios, described in Sect. 3.1.

The ratio $R_{B / S}$ for the model moccA-39 represents the simplest case. It is equal to the ratio between the number of EMT dived by the number of EM BSs (no dynamical BSs). The blue line in Fig. 7 ( $R_{B / S}$ consists of all channels) is very much the same as in the top panel of Fig. 8 where $R_{B / S}^{\text {evol }}$ consists of only EMT and EM. The number of dynamical BSs is low, thus, the ratio $R_{B / S}^{d y n}$ has a large 


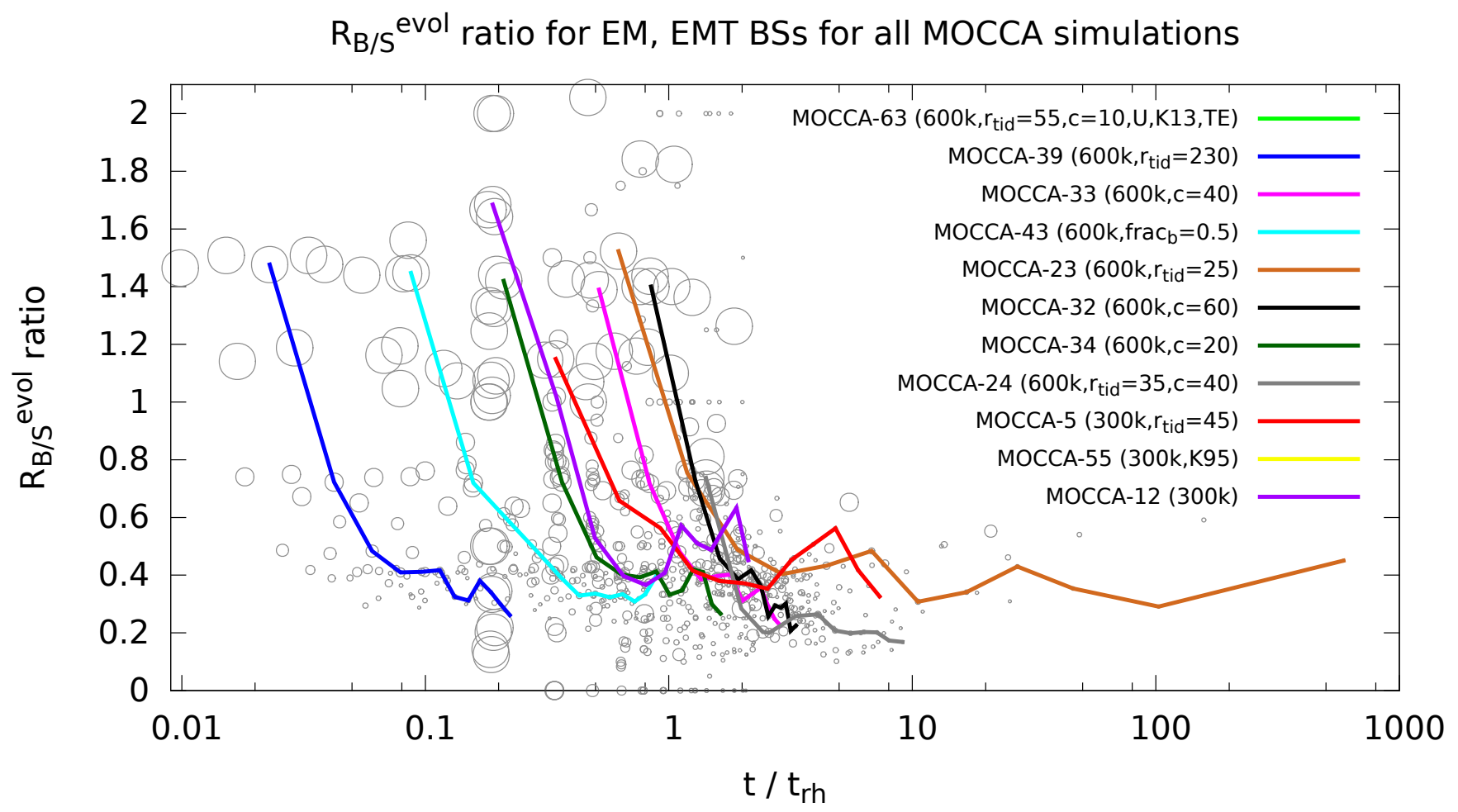

$\mathrm{R}_{\mathrm{B} / \mathrm{S}}$ dyn ratio for dynamical BSs for all MOCCA simulations

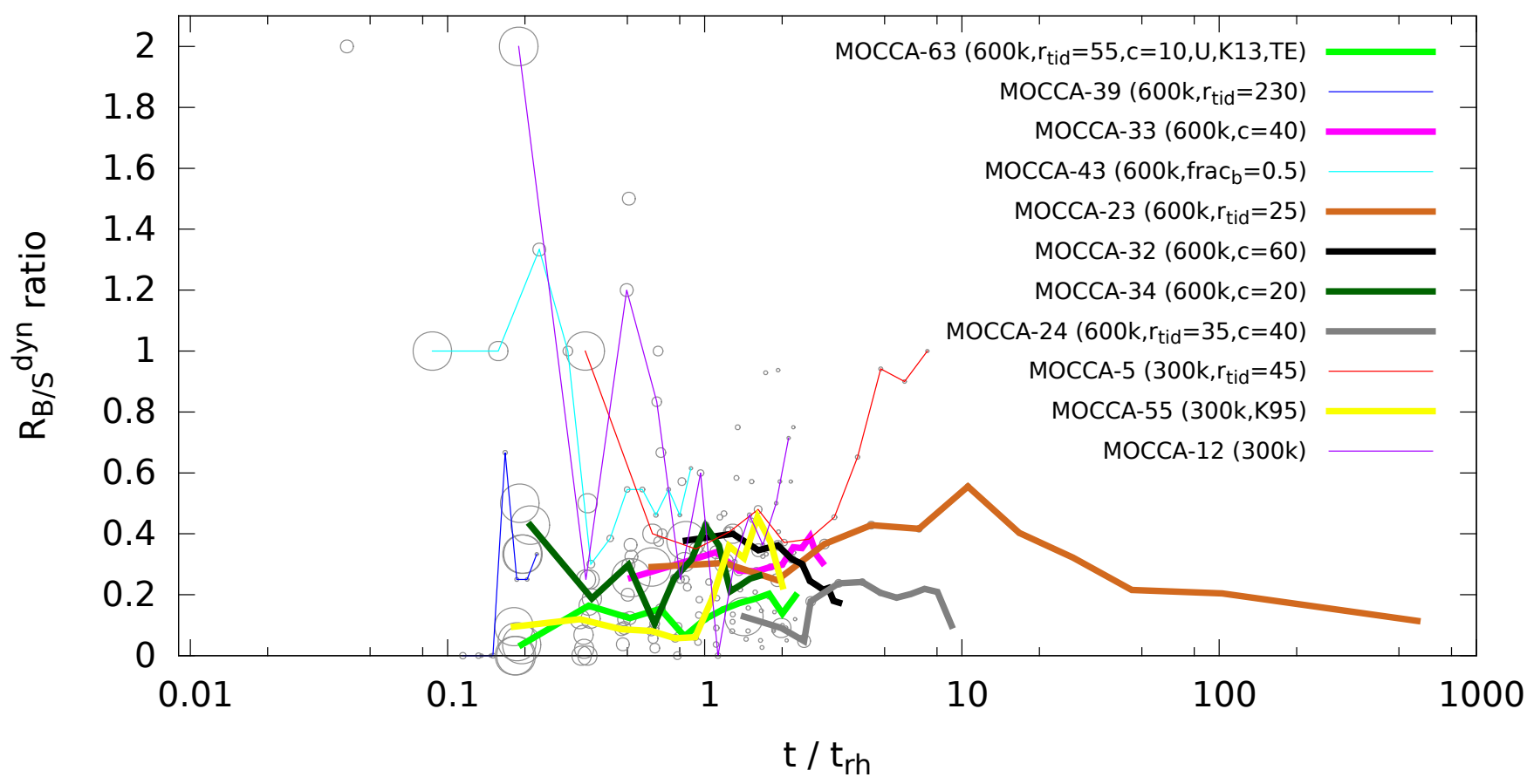

Figure 8. Evolving ratios between BSs in binaries and as single stars for all MoccA simulations described in Sect. 2.1 for evolutionary (top, $R_{B / S}^{\text {evol }}$ ) and dynamical (bottom, $R_{B / S}^{d y n}$ ) BSs separately. The meaning of symbols and lines is the same as for Fig. 7. The colors of lines for selected models are consistent with Fig. 7 too. For the top plot two models (MoccA-63, MoccA-55) are not presented because of the low number of EM and EMT BSs (around 10) and thus a large scatter. For dynamical BSs (bottom panel) not all simulations are plotted (thus, a lower number of gray circles). Thin lines in the bottom plot indicate the models with a very low number of dynamical BSs $(\sim 10)$ which introduce a large scattering. For details see the text. 
scatter (see bottom panel in Fig. 8). The model is dominated by evolutionary BSs. Nevertheless, the overall ratio $R_{B / S}$ stays around the value 0.3 .

\subsubsection{MOCCA-43}

The ratio $R_{B / S}$ for the model moccA-43 (light blue line in Fig. 7) evolves in a similar way as in Mocca-39. The model MoccA-43 has $50 \%$ of primordial binaries, whereas мосса- 39 has $20 \%$. The evolution of the ratio $R_{B / S}$ follows the evolution of the ratio $R_{B / S}^{\text {evol }}$ too (see Fig. 8). For model MoccA-43 only the number of BSs is larger, but the overall trend of the number of EM and EMT is very much the same as for MoccA-39. The number of dynamical BSs is slightly larger, but still very low. Thus, the ratio $R_{B / S}^{d y n}$ scatter a lot too (see Fig. 8).

\subsubsection{MOCCA-34, MOCCA-33}

Other examples of the MOCCA-DROPPING group are models MOcCA-34 (dark green) and MоссA-33 (magenta). These are the models which are initially more concentrated than previously discussed моссA-39 model (dark blue). The rest of the initial conditions are the same. The number of EM and EMT BSs is the same for these models despite the higher concentrations (see Fig. 9). This is also the reason why the ratio $R_{B / S}^{\text {evol }}$ follows closely the ratio $R_{B / S}$ (see Fig. 8). This is another example which shows that the concentration itself does not change the population of the evolutionary BSs (more details on the influence of the concentration on the population of BSs of different types one can find in Sect. 3.2.4).

However, the higher concentrations cause that the number of dynamical BSs becomes significant for models mocca-34 and мосса-33. Interestingly, a higher number of dynamical BSs does not change the overall ratio $R_{B / S}$. Both, $R_{B / S}^{d y n}$ and $R_{B / S}^{\text {evol }}$, oscillate around value 0.3 (see Fig. 8)

\subsubsection{MOCСА-32, MOССА-23, MOCСA-24}

The ratio $R_{B / S}$ drops below 0.3 for models which are very dense or close to dissolution.

MOCCA-32 is an example of a very concentrated model. It starts with $600 \mathrm{k}$ stars and concentration $c=r_{\text {tid }} / r_{h}=60$. It means that half of the GC's mass is contained in the radius $r_{h}=1.7$ [pc] (see Tab. 1). This very large initial concentration causes that the number of dynamical BSs is very high right from the beginning (see Fig. 9).

Another model, MoccA-23, starts with 600k stars, but with $r_{\text {tid }}$, thus $c=25 / 10=2.5[\mathrm{pc}]$. This model dissolves completely within 12 Gyr (see Fig. 9).

The next model, MOccA-24, is even denser. It starts with $600 \mathrm{k}$ stars, $r_{\text {tid }}=35$ and $c=40$, thus $r_{h}=0.9$ [pc]. Because of the larger $r_{\text {tid }}$, the model takes a litte bit more time for the complete dissolution. The number of dynamical BSs for the mocca-24 is dominant right from the beginning. The high density of the GC causes that the probabilities of the dynamical interactions are higher than for other models. Simultaneously, it causes that within a few first Gyr many of the binaries are disrupted (half of the binaries in the first $900 \mathrm{Myr}$ ). Thus, the number of dynamical BSs drops after 2 Gyr to a level around 100 BSs (see Fig. 9). Then, the EM channel starts to be the dominant one, like for other models with similar initial conditions.

It is worth to notice that for such a dense GC a smaller $r_{\text {tid }}$ and a high concentration start to make a difference in the number of EM and EMT BSs. The number of EM and EMT for MOcCA24 models is clearly smaller than in MoccA- 23 or MoccA- 32 models despite the same initial number of primordial binaries and the same semi-major axes distribution (for details see Sect. 3.2.4). For these three models (MOсса-32, MOсса-23, MOсCA-24) the dynamical scales of the GC evolution are larger than in other models. For mocca-23, i.e., the one which dissolves just after $12 \mathrm{Gyr}$, the dynamical times exceeds hundreds of the half-max relaxation times ( $t_{r h}$, see Fig. 7).

The ratio $R_{B / S}$ drops below 0.3 for models MoccA-32, MoccA24 , and mocca-23. The lower ratio $R_{B / S}$ is a consequence of the low number of EMT BSs, or increased number of DBS+DBB, or both. Fig. 9 shows that for MoccA-32 model the number of dissolved BSs (DBS+DBB) raises after the first few Gyr and stays important until 12 Gyr. This is caused by the very high concentration of the GC which highly increases probabilities for strong dynamical interactions which will disrupt a binary. It causes that some of BSs become single stars. Thus, the ratio $R_{B / S}^{d y n}$ is consequently dropping from around $R_{B / S}^{d y n} \sim 0.4$ to $R_{B / S}^{d y n}<0.2$ at $12 \mathrm{Gyr}$ (see the bottom plot in Fig. 8). Additionally, the $R_{B / S}^{e v o l}$ is also consequently dropping for later times too and goes slightly below the level 0.3 (see the top panel in Fig. 8). The ratio $R_{B / S}^{\text {evol }}$ is getting lower with time because the number of EMT is getting lower. The high concentration of the Mocca-32 model destroyed some binaries which would otherwise create EMT BSs if their evolution was unperturbed (like e.g. in model MoccA-39). It concerns mainly wide EMT BSs which are formed through the stellar winds. They are easier to disrupt than tight EMT. However, the number of EM for моссA-32 is similar as for the slowly evolving MoccA-39 model (see Fig. 8). They are formed in close binaries, which are much harder to be disrupted due to dynamical interactions even in the dense clusters.

For the MoccA-24 model the ratios $R_{B / S}^{\text {evol }}, R_{B / S}^{d y n}$ and $R_{B / S}$ drops below 0.3 due to the same reasons as for the moccA-32 model. Additionally, моссA- 24 has even a smaller tidal radius, thus, the density of this cluster is even higher. Due to a higher density there are even more binaries destroyed because of strong dynamical interactions. As a result, the number of EMT BSs is even smaller for this model (see Fig. 9). It causes that the ratio $R_{B / S}^{\text {evol }}$ goes below 0.3 just after $2 t_{r h}$ (see Fig. 8). The number of DBS+DBB BSs is marginal - for such a dense GC, the binaries with BSs are hard and thus difficult to destroy due to dynamical interactions. Hence, the number of dissolved binaries does not influence the low ratio $R_{B / S}^{d y n}$. The number of CSS BSs is around 10 (formed in collisions due to dynamical interactions between two single stars). However, their number is too small to make the ratio $R_{B / S}^{d y n}$ significantly lower. The only explanation is that dynamical interactions preferentially create BSs as single stars. It has to be connected with the high density of the Mocca-24 model. However, the exact reason for that still has to be found. The ratio $R_{B / S}^{d y n}$ is consequently lower than 0.3 (see Fig. 8).

The mocca-23 is also a model for which the ratio $R_{B / S}$ drops below 0.3 . It is a very fast evolving model, because it has small $r_{\text {tid }}=25$ [pc]. For the default concentration $c=10$ the half-mass radius is small $r_{h}=2.5$ [pc], which makes it also a very dense GC. It needs only $12 \mathrm{Gyr}$ for the complete dissolution (see Fig. 9). The ratio $R_{B / S}^{\text {evol }}$ for this model oscillates around 0.4 and does not go below 0.3 , like for previously discussed models. The ratio $R_{B / S}$ drops below 0.3 because there is a high number of DBS+DBB BSs for this model (see Fig. 9). 

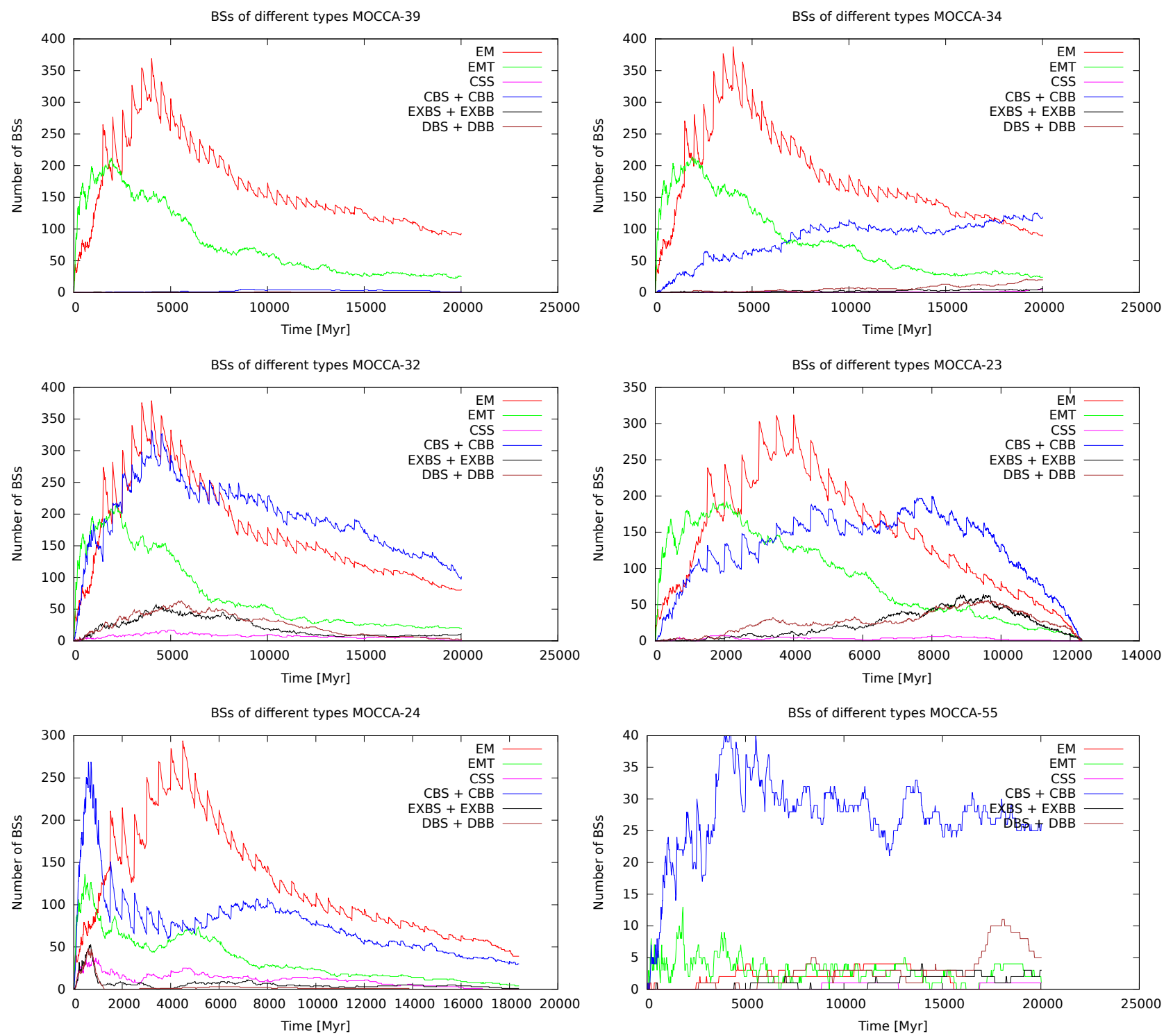

BSs of different types MOCCA-12

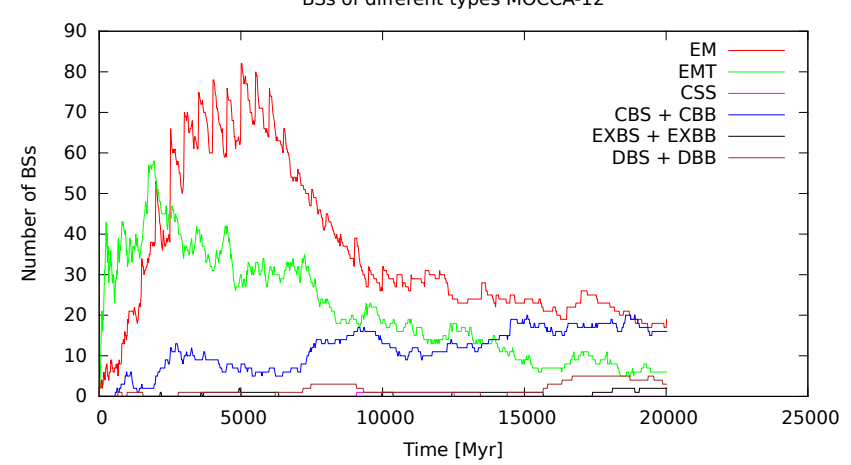

Figure 9. Population of BSs of different types for selected models. Types of BSs are described in Sect. 3.1. The names of models are given in the titles of the plots. The parameters of models are summarized in Tab. 2 and Tab. 2.

\subsubsection{MOсCA-5, MOCCA-12}

The models MoccA-5 (red), MoccA-12 (violet) are examples which show some clear peak in the ratio $R_{B / S}$ for the dynamical times larger than $1 t_{r h}$ (see Fig. 7). They have a low number of dynamical
BSs, thus the ratio $R_{B / S}^{d y n}$ scatter a lot (see Fig. 8). The number of EMT is also low ( $\sim 10 \mathrm{BSs}$ ), thus the ratio $R_{B / S}^{\text {evol }}$ scatter too (see Fig. 8). As a result, the ratio $R_{B / S}$ scatter when a GC becomes dynamically old ( $>$ a few of $t_{r h}$ ). 

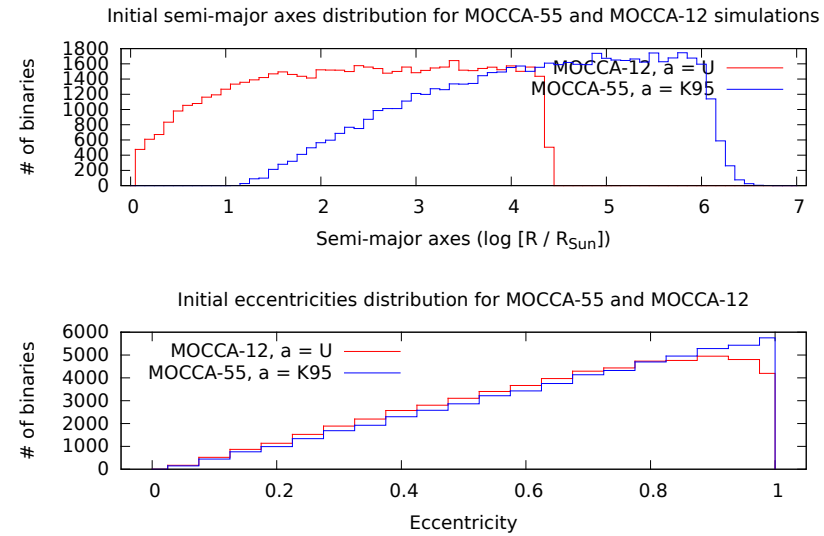

Figure 10. Initial distributions of semi-major axes (top) and eccentricities (bottom) for models MOCCA-55 and MoccA-12. The first model is a representative of the MocCA-RAISING group, whereas the latter one is an example of MOCCA-DROPPING group (see Sect. 3.3). For the meaning of the symbols see Tab. 2, for the number of BSs of these models see Fig. 9, for details see text.

\subsubsection{Models from MOcCA-RAISING group}

The group Mocca-RAISING consists of the models which start with low ratios $R_{B / S}$ (see Fig. 7). It is a result of a different initial semimajor axes distributions of binaries in comparison to models of the MOCCA-DROPPING group.

The model MoccA-55 is an example of the MoccA-RAISING group (yellow line in Fig. 7 and Fig. 8). It starts with 300k stars, with $r_{\text {tid }}=$ 69 [pc] and concentration $c=10$ (see Tab. 2). The initial semimajor axes distribution is created according to Kroupa (1995a).

Fig. 10 presents a comparison of the initial conditions between models MoсcA-55 and MоссA-12. The latter one is an example from the MOCCA-DROPPING group. They differ in only one initial parameter (see Tab. 1, Tab. 2). The initial semi-major axes distributions are compared on the top panel of Fig. 10, while distributions of eccentricities on the bottom panel. For MoccA-55 there are many more wide binaries, whereas the eccentricity distribution is very much the same. However, there are small differences for high eccentricities $(>0.9)$. It is caused by the fact that the mocca code, while generating the initial conditions, checks for immediate mergers. It may happen that for a compact binary a high eccentricity will be drawn. For such binary the periastron distance could be smaller than the sum of radii of the system components. The binary would merge just in the first call of the stellar evolution. In order to avoid such situation the мосса code generates the eccentricity for the binary once again. For the моссA-12 there are many more compact binaries, thus this situation may happen more often. As a result, there is slightly less high eccentricities for this model (see bottom panel in Fig. 10). All other initial conditions for the MocCA-55 and моссA-12 models are the same (see Tab. 1 and Tab. 2).

Fig. 11 shows the density maps which combines distributions of semi-major axes with eccentricities for the model MoсcA55 (MOCCA-RAISING). The top panel shows the initial density map, whereas the bottom shows the density for $12 \mathrm{Gyr}$. The bottom plot consists of only the binaries with main sequence stars. These are the types of objects which still have a chance to create BSs in a physical collision (CBS, CBB), due to mass transfer when one of the stars leaves main-sequence (EMT) or due to a merger (EM). Fig. 11 shows that for this model there are many wide binaries $\left(a>10^{4}\left[R_{\odot}\right]\right)$, also with high eccentricities for time $T=0$. After 12 Gyr there are almost no binaries with semi-major axes
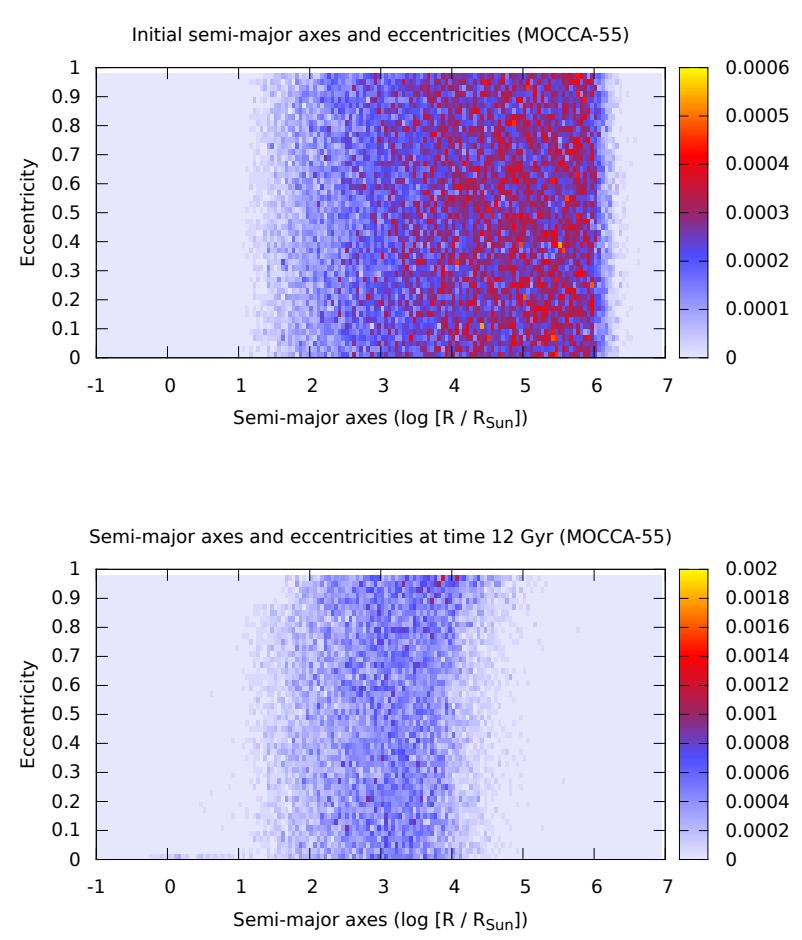

Figure 11. Density maps of the semi-major axes - eccentricity distribution for $T=0$ (top) and for time $12 \mathrm{Gyr}$ (bottom) for the model MoccA-55. For 12 Gyr the density map is produced only for MS-MS binaries. For the meaning of the symbols see Tab. 2, for the number of BSs of these models see Fig. 9, for details see text.

$>10^{4}\left[R_{\odot}\right]$. However, there are still these $\lesssim 10^{4}\left[R_{\odot}\right]$ with high eccentricities $(\approx 1)$.

The differences in the initial distributions of semi-major axes in binaries (see Fig. 10) cause the differences in the number of BSs of various types for these models (see Fig. 9). For moccA-12 model, which represents the group MOCCA-DROPPING, the dominant channels from the beginning are EM and EMT (evolutionary BSs). The number of dynamical BSs increases with time because GC is getting denser. In turn, MOcCA-55 has a very low number of EM and EMT and a high number of dynamical BSs.

The differences in the number of BSs of different populations between the models MoсcA-55 and MоссA-12 is due to the different frequency of dynamical interactions and mergers. Fig. 12 shows on the top panel the number of the dynamical interactions between binaries and single stars and between two binaries. The bottom panel in Fig. 12 shows the number of evolutionary mergers and physical collisions between stars due to dynamical interactions.

The low number of the dynamical BSs in MoccA-12 from MOCCA-DROPPING group (see Fig. 9) is caused by the low number of the dynamical interactions (only $\sim 20 k$ up to $12 \mathrm{Gyr}$, see Fig. 12). Thus, the number of collisions due to dynamical interactions is also low. After 12 Gyr only a few dozens of stars collided (violet and light blue line on the bottom panel in Fig. 12). Instead, the моссA12 model has many mergers due to stellar evolution (black line). This is a result of the initial semi-major axes distribution. For the model MoccA-12 there are many compact binaries which create EM and EMT BSs. Dynamical BSs become significant only later, when the density of the GC increases and the cluster slowly goes to the core collapse. 

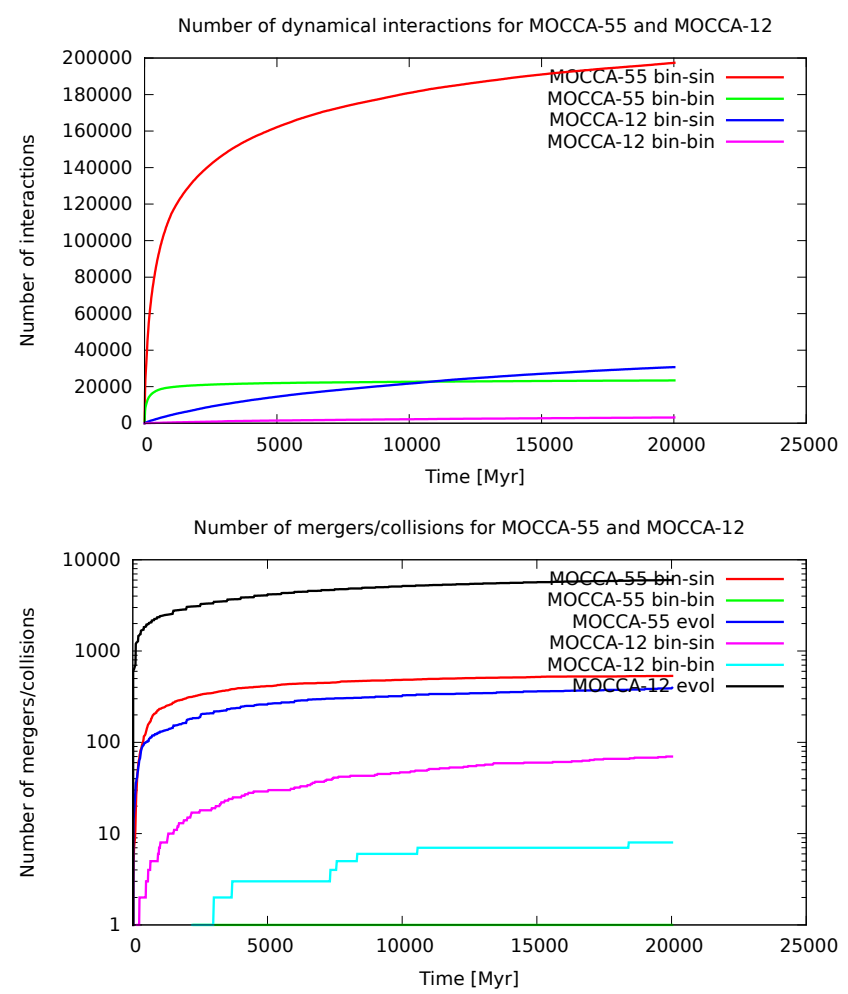

Figure 12. Number of dynamical interactions (top panel), mergers and collisions (bottom) for models MoсcA-55 and Mocca-12. The term bin-sin stands for a dynamical interaction between a binary and a single star, the term bin-bin for a dynamical interaction between two binaries, the term merger for coalescence between two components in a binary without involving any sort of a dynamical interaction. The merger is a result of the stellar evolution only. The term collision concerns a physical collision between two or more stars due to a dynamical interaction. The моссA-55 model represents the group MocCA-RAISING, whereas MoccA-12 the group MOCCA-DROPPING. For details see the text.

The model Mocca-55 which represents MoccA-RAISING group has a low number of EM and EMT (see Fig. 9). For this model there is a small number of compact binaries (see Fig. 10) and thus there are not many binaries which could possibly create evolutionary BSs. Instead, the number of the dynamical BSs is high. The number of CBS and $\mathrm{CBB}$ increases from the beginning and it is important throughout the whole GC evolution.

The number of dynamical interactions for MOсCA-55 is around 10 times larger than for the мосса-12. The high number of the interactions is a result of larger semi-major axes for binaries (see Fig. 10). It increases probabilities of interactions. Many of them are just fly-by passages between objects. They do not create large differences in semi-major axes of binaries. However, due to a large number of them the eccentricity of some binaries increases (even to $>0.99$ ). The increased eccentricities cause that the code detects a collision when the periastron distance is smaller than the sum of radii of stars and the binary collides. This is not a typical collision between two stars in a dynamical interaction. The incoming star or binary does not actually collide with the binary, instead it changes only its properties. As a result of this interaction the collided star is a single star. Thus, the ratio $R_{B / S}$ for the MOcCA-RAISING group of models starts with small values $R_{B / S} \lesssim 0.3$ (see Fig. 7).

A large number of dynamical interactions disrupts many very wide binaries (MOCCA-55). As a result, after 12 Gyr there is actually a few of them left (see Fig. 11). Because the number of wide binaries with high eccentricities is getting lower with time, also the number of the dynamical BSs created from wide binaries with high eccentricities is getting lower. More dynamical BSs are created in physical collisions between a star in a binary and some incoming star (or binary). As a result BSs stay in binaries. Thus, the ratio $R_{B / S}^{d y n}$ slowly increases with time (see Fig. 8). Additionally, the $R_{B / S}$ which combines all BSs from all channels increases too (see Fig. 7). The number of EM and EMT BSs is low during the whole simulation, thus the ratio $R_{B / S}^{\text {evol }}$ scatter a lot. However, it has a small influence on the ratio $R_{B / S}$. Dynamical BSs, after wide high-eccentricity binaries are disrupted, are created in the same way as BSs from the MOCCA-DROPPING group. Thus, the ratio $R_{B / S}$ from MOCCA-RAISING group reaches the same values as the models of the MOCCA-DROPPING group.

\section{SUMMARY}

Blue stragglers (BSs) are very interesting stars in terms of their formation and evolution. Their increased mass indicates that they have received an additional mass during their life. The main channels of their formation concern the stellar evolution (mass gained through the mass transfer) and the dynamical interactions (mass gained due to physical collisions). BSs are more numerous in globular clusters than in the field of the Galaxy. This make the BSs in GCs extremely interesting objects to study the complex interplay between the stellar evolution and the dynamical evolution of GCs.

Initial conditions of GCs have a fundamental influence on populations of BSs of different types. It concerns both, the global initial parameters of a GCs, and the initial distributions of binaries properties. Different initial conditions can very significantly change populations of BSs.

In this context the importance of the semi-major axes distribution is crucial. The high number of compact binaries, like for the distribution $a=U L$ (see Fig. 2 and definitions in Tab. 1), directly relates to the high number of EM and EMT BSs. When the initial number of compact binaries is lower, like for $a=L$ distribution, the number of EM and EMT decreases (see Fig. 2). It decreases even more for distributions like K95 (see Fig. 3) which prefer even wider binaries.

The influence of the semi-major axes distribution on the population of EM and EMT is a consequence of the formation scenarios of these BSs. EM are formed in compact binaries. Thus, when the initial semi-major axis is wider, the binary needs much more time to merge. The same applies for the first subgroup of EMT which are also compact binaries (see Sect. 3.1). For them a large semi-major axes prevent the mass transfer as well. For the second subgroup of EMT the semi-major axes can be larger, but a high eccentricity is needed to make the mass transfer possible. However, the number of EMT created according to this scenario is rather low. Thus, it has a smaller impact on the overall population of EMT.

It was very unexpected to find out that a large number of wide binaries can so significantly influence the population of dynamical BSs (CBS, CBB, see Fig. 3). In this mechanism wider binaries have higher probabilities of dynamical interactions. These interactions are mostly fly-bys but increase the eccentricities of many binaries. For a number of them the eccentricities get so large that a collision takes place and the binary merges. In this scenario, because of a large number of semi-major axes with wide binaries, the dynamical BSs are the dominant ones.

The influence of the initial semi-major axes distributions on 
populations of BSs can be very valuable for narrowing down the initial conditions. If a good way to distinguish between evolutionary and the dynamical BSs is found, it could help to give some boundaries on the initial semi-major axes distribution - it is a very important subject in the studies of GCs.

Higher initial concentrations of GCs have a large impact on the population of dynamical BSs. The number of CBS and CBB $\mathrm{BSs}$ increase for larger concentrations. It is a consequence of higher probabilities of strong dynamical interactions for denser systems. If the density increased, the number of dynamical BSs changing their types increases too. There are more binaries which exchange their companions (EXBS, EXBB) or are dissolved (DBS, DBB) due to strong dynamical interactions.

Surprisingly, higher initial concentrations of GCs do not have any influence on the population of evolutionary BSs. For the моссA7 model the concentration is $c=r_{\text {tid }} / r_{h}=40$, which means that half of the GC's mass is contained inside $r_{h}=1.7$ [pc]. This is a very dense model and even for such extreme conditions populations of EM and EMT BSs were not affected noticeably. It has very important implications for observations. It strongly supports the theory that evolutionary BSs are results of the unperturbed evolution of the primordial binaries. The same applies even if the concentration is increased up to $c=60-$ the population of EM and EMT BSs is also not affected.

Different initial conditions have also a profound effect on the ratio between $\mathrm{BSs}$ in binaries and as single stars $\left(R_{B / S}\right)$. The моссA models from Tab. 1 and Tab. 2 vary in many aspects. They have various initial concentrations which implies different dynamical ages for GCs. They have also various initial semi-major axes and eccentricities distributions. Surprisingly, the ratio $R_{B / S}$ for all of the models gets close to the value $R_{B / S} \sim 0.4$ within a few Gyrs.

There are two groups which have different evolutions of the ratio $R_{B / S}$. The first group, MOcCA-DropPING, concerns the models which have some fraction of compact binaries. For them, the channels EM are EMT are important. Their ratio $R_{B / S}$ drops from the values $\gtrsim 1.0$ to around 0.4 . The second group, Mocca-RAISING, consists of models which have a large fraction of wide binaries. For them the EM and EMT channels are almost not important, whereas dynamical BSs are the dominant ones. The ratio $R_{B / S}$ for moccARAISING starts with low values and raises with time to reach $\sim 0.4$. It is very interesting that the main reason behind this division is the initial semi-major axes distribution. The initial concentrations of GCs do not have a significant influence on these two groups of models.

However, an attempt to understand why the ratio $R_{B / S}$ oscillates around the value 0.4 for such a variety of different initial conditions still has to be determined. It only suggests that, because of some reason, $\sim 40 \%$ of all dynamical interactions lead to the creation of binaries, and the rest to the dissolution of binaries.

\section{ACKNOWLEDGMENT}

The project was supported partially by Polish National Science Center grants DEC-2011/01/N/ST9/06000 and DEC2012/07/B/ST9/04412.

\section{REFERENCES}

Bragaglia A., Carretta E., Blanco A. R., Cacciari C., Kinman T. D., 2005, in Hill V., François P., Primas F., eds, From Lithium to Uranium: Elemental Tracers of Early Cosmic Evolution Vol. 228 of IAU Symposium,
Chemical properties of field halo and thick disk blue straggler stars: first results. pp 243-244

Casertano S., Hut P., 1985, ApJ, 298, 80

Clarkson W. I., Sahu K. C., Anderson J., Rich R. M., Smith T. E., Brown T. M., Bond H. E., Livio M., Minniti D., Renzini A., Zoccali M., 2011, ApJ, 735, 37

Davies M. B., Piotto G., de Angeli F., 2004, MNRAS, 349, 129

De Marco O., Shara M. M., Zurek D., Ouellette J. A., Lanz T., Saffer R. A., Sepinsky J. F., 2005, ApJ, 632, 894

Dotter A., Sarajedini A., Anderson J., Aparicio A., Bedin L. R., Chaboyer B., Majewski S., Marín-Franch A., Milone A., Paust N., Piotto G., Reid I. N., Rosenberg A., Siegel M., 2010, ApJ, 708, 698

Ferraro F. R., Beccari G., Dalessandro E., Lanzoni B., Sills A., Rood R. T., Pecci F. F., Karakas A. I., Miocchi P., Bovinelli S., 2009, Nature, 462, 1028

Ferraro F. R., Fusi Pecci F., Bellazzini M., 1995, A\&A, 294, 80

Ferraro F. R., Lanzoni B., 2009, in Revista Mexicana de Astronomia y Astrofisica Conference Series Vol. 37 of Revista Mexicana de Astronomia y Astrofisica Conference Series, Probing the link between dynamics and stellar evolution: Blue Straggler Stars in Globular clusters. pp 6271

Ferraro F. R., Messineo M., Fusi Pecci F., de Palo M. A., Straniero O., Chieffi A., Limongi M., 1999, AJ, 118, 1738

Ferraro F. R., Paltrinieri B., Fusi Pecci F., Cacciari C., Dorman B., Rood R. T., Buonanno R., Corsi C. E., Burgarella D., Laget M., 1997, A\&A, 324,915

Ferraro F. R., Sabbi E., Gratton R., Piotto G., Lanzoni B., Carretta E., Rood R. T., Sills A., Fusi Pecci F., Moehler S., Beccari G., Lucatello S., Compagni N., 2006, ApJ, 647, L53

Fiorentino G., Lanzoni B., Dalessandro E., Ferraro F. R., Bono G., Marconi M., 2014, ApJ, 783, 34

Fossati L., Mochnacki S., Landstreet J., Weiss W., 2010, A\&A, 510, A8

Fregeau J. M., Rasio F. A., 2007, ApJ, 658, 1047

Fuhrmann K., Chini R., Hoffmeister V. H., Stahl O., 2011, MNRAS, 416, 391

Fusi Pecci F., Ferraro F. R., Corsi C. E., Cacciari C., Buonanno R., 1992, AJ, 104, 1831

Geller A. M., Mathieu R. D., 2011, Nature, 478, 356

Geller A. M., Mathieu R. D., 2012, AJ, 144, 54

Giersz M., 1998, MNRAS, 298, 1239

Giersz M., 2001, MNRAS, 324, 218

Giersz M., 2006, MNRAS, 371, 484

Giersz M., Heggie D. C., 1994a, MNRAS, 268, 257

Giersz M., Heggie D. C., 1994b, MNRAS, 270, 298

Giersz M., Heggie D. C., 1996, MNRAS, 279, 1037

Giersz M., Heggie D. C., 1997, MNRAS, 286, 709

Giersz M., Heggie D. C., Hurley J., Hypki A., 2011, ArXiv e-prints

Giersz M., Heggie D. C., Hurley J. R., Hypki A., 2013, MNRAS, 431, 2184

Giersz M., Spurzem R., 1994, MNRAS, 269, 241

Harris W. E., 1996, AJ, 112, 1487

Heggie D. C., 1975, MNRAS, 173, 729

Hénon M. H., 1971, Ap\&SS, 14, 151

Hills J. G., Day C. A., 1976, Astrophys. Lett., 17, 87

Hurley J. R., Pols O. R., Tout C. A., 2000, MNRAS, 315, 543

Hurley J. R., Tout C. A., Pols O. R., 2002, MNRAS, 329, 897

Hypki A., Giersz M., 2013, MNRAS, 429, 1221

Kaluzny J., Rozyczka M., Thompson I. B., Zloczewski K., 2009, Acta Astron., 59, 371

Kaluzny J., Rucinski S. M., Thompson I. B., Pych W., Krzeminski W., 2007, AJ, 133, 2457

Kaluzny J., Thompson I. B., Rucinski S. M., Pych W., Stachowski G., Krzeminski W., Burley G. S., 2007, AJ, 134, 541

Knigge C., Leigh N., Sills A., 2009, Nature, 457, 288

Kroupa P., 1995a, MNRAS, 277, 1491

Kroupa P., 1995b, MNRAS, 277, 1507

Kroupa P., Gilmore G., Tout C. A., 1991, MNRAS, 251, 293

Kroupa P., Tout C. A., Gilmore G., 1993, MNRAS, 262, 545 
Kroupa P., Weidner C., Pflamm-Altenburg J., Thies I., Dabringhausen J., Marks M., Maschberger T., 2013, The Stellar and Sub-Stellar Initial Mass Function of Simple and Composite Populations. p. 115

Lanzoni B., Sanna N., Ferraro F. R., Valenti E., Beccari G., Schiavon R. P., Rood R. T., Mapelli M., Sigurdsson S., 2007, ApJ, 663, 1040

Lee J.-W., Carney B. W., 2002, AJ, 124, 1511

Leigh N., Sills A., Knigge C., 2007, ApJ, 661, 210

Leigh N., Sills A., Knigge C., 2008, in E. Vesperini, M. Giersz, \& A. Sills ed., IAU Symposium Vol. 246 of IAU Symposium, Where the Blue Stragglers Roam: Searching for a Link Between Formation and Environment. pp 331-335

Mapelli M., Ripamonti E., Tolstoy E., Sigurdsson S., Irwin M. J., Battaglia G., 2007, MNRAS, 380, 1127

Mapelli M., Sigurdsson S., Ferraro F. R., Colpi M., Possenti A., Lanzoni B., 2006, MNRAS, 373, 361

Mardling R. A., Aarseth S. J., 2001, MNRAS, 321, 398

Mateo M., Fischer P., Krzeminski W., 1995, AJ, 110, 2166

Mateo M., Harris H. C., Nemec J., Olszewski E. W., 1990, AJ, 100, 469

Mathieu R. D., Geller A. M., 2009, Nature, 462, 1032

McCrea W. H., 1964, MNRAS, 128, 147

Milone A. P., Piotto G., Bedin L. R., Sarajedini A., 2008, Mem. Soc. Astron. Italiana, 79, 623

Monelli M., Cassisi S., Mapelli M., Bernard E. J., Aparicio A., Skillman E. D., Stetson P. B., Gallart C., Hidalgo S. L., Mayer L., Tolstoy E., 2012, ApJ, 744, 157

Otulakowska M., Olech A., Pych W., Pamyatnykh A. A., Zdravkov T., Rucinski S. M., 2011, Acta Astron., 61, 161

Piotto G., De Angeli F., King I. R., Djorgovski S. G., Bono G., Cassisi S., Meylan G., Recio-Blanco A., Rich R. M., Davies M. B., 2004, ApJ, 604, L109

Pritchet C. J., Glaspey J. W., 1991, ApJ, 373, 105

Pych W., Kaluzny J., Krzeminski W., Schwarzenberg-Czerny A., Thompson I. B., 2001, A\&A, 367, 148

Sandage A. R., 1953, AJ, 58, 61

Santolamazza P., Marconi M., Bono G., Caputo F., Cassisi S., Gilliland R. L., 2001, ApJ, 554, 1124

Shara M. M., Saffer R. A., Livio M., 1997, ApJ, 489, L59

Sollima A., Lanzoni B., Beccari G., Ferraro F. R., Fusi Pecci F., 2008, A\&A, 481,701

Stodolkiewicz J. S., 1986, Acta Astron., 36, 19

Thompson I. B., Kaluzny J., Rucinski S. M., Krzeminski W., Pych W., Dotter A., Burley G. S., 2010, AJ, 139, 329

Tillich A., Przybilla N., Scholz R.-D., Heber U., 2010, A\&A, 517, A36

Wang L., Spurzem R., Aarseth S., Giersz M., Askar A., Berczik P., Naab T., Schadow R., Kouwenhoven M. B. N., 2016, MNRAS, 458, 1450

Zinn R., Searle L., 1976, ApJ, 209, 734

This paper has been typeset from a $\mathrm{T}_{\mathrm{E}} \mathrm{X} / \mathrm{L}_{\mathrm{E}} \mathrm{X}$ file prepared by the author. 